\title{
Statistical study of the location and size of the electron edge of the Low-Latitude Boundary Layer as observed by Cluster at mid-altitudes
}

\author{
Y. V. Bogdanova ${ }^{1}$, C. J. Owen ${ }^{1}$, A. N. Fazakerley ${ }^{1}$, B. Klecker ${ }^{2}$, and H. Rème ${ }^{3}$ \\ ${ }^{1}$ Mullard Space Science Laboratory, University College London, Holmbury St. Mary, Dorking RH5 6NT, UK \\ ${ }^{2}$ MPI für Extraterrestrische Physik, Giessenbachstrasse, 85748 Garching, Germany \\ ${ }^{3}$ Centre d'Etude Spatiale des Rayonnements, 31028 Toulouse Cedex 4, France
}

Received: 29 March 2006 - Revised: 21 July 2006 - Accepted: 24 August 2006 - Published: 20 October 2006

\begin{abstract}
The nature of particle precipitations at dayside mid-altitudes can be interpreted in terms of the evolution of reconnected field lines. Due to the difference between electron and ion parallel velocities, two distinct boundary layers should be observed at mid-altitudes between the boundary between open and closed field lines and the injections in the cusp proper. At lowest latitudes, the electrondominated boundary layer, named the "electron edge" of the Low-Latitude Boundary Layer (LLBL), contains softmagnetosheath electrons but only high-energy ions of plasma sheet origin. A second layer, the LLBL proper, is a mixture of both ions and electrons with characteristic magnetosheath energies. The Cluster spacecraft frequently observe these two boundary layers. We present an illustrative example of a Cluster mid-altitude cusp crossing with an extended electron edge of the LLBL. This electron edge contains 10$200 \mathrm{eV}$, low-density, isotropic electrons, presumably originating from the solar wind halo population. These are occasionally observed with bursts of parallel and/or anti-paralleldirected electron beams with higher fluxes, which are possibly accelerated near the magnetopause X-line. We then use 3 years of data from mid-altitude cusp crossings ( 327 events) to carry out a statistical study of the location and size of the electron edge of the LLBL. We find that the equatorward boundary of the LLBL electron edge is observed at 10:00-17:00 magnetic local time (MLT) and is located typically between $68^{\circ}$ and $80^{\circ}$ invariant latitude (ILAT). The location of the electron edge shows a weak, but significant, dependence on some of the external parameters (solar wind pressure, and IMF $B_{Z^{-}}$component), in agreement with expectations from previous studies of the cusp location. The latitudinal extent of the electron edge has been estimated using new multi-spacecraft techniques. The Cluster tetrahedron crosses the electron and ion boundaries of the LLBL/cusp
\end{abstract}

Correspondence to: $\mathrm{Y} . \mathrm{V}$. Bogdanova

(jb@mssl.ucl.ac.uk) with time delays of 1-40 min between spacecraft. We reconstruct the motion of the electron boundary between observations by different spacecraft to improve the accuracy of the estimation of the boundary layer size. In our study, the LLBL electron edge is distinctly observed in $87 \%$ of mid-altitude LLBL/cusp crossings with clear electron and ion equatorward boundaries equivalent to $35 \%$ of all LLBL/cusp crossings by Cluster. The size of this region varied between $0^{\circ}-2^{\circ}$ ILAT with a median value of $0.2^{\circ}$ ILAT. Generally, the size of the LLBL electron edge depends on the combination of many parameters. However, we find an anti-correlation between the size of this region and the strength of the IMF, the absolute values of the IMF $B_{Y}$ - and $B_{Z}$-components and the solar wind dynamic pressure, as is expected from a simple reconnection model for the origin of this region.

Keywords. Magnetospheric physics (Magnetopause, cusp, arid boundary layers; Solar wind-magnetosphere interactions) - Space plasma physics (Magnetic reconnection)

\section{Introduction}

The Earth's cusps were first discussed by Chapman and Ferraro (1931) as two magnetic null points, one in each hemisphere. It was suggested that these nulls would be points of solar wind plasma entry into the magnetosphere. Later, satellite observations revealed the existence of magnetosheathtype plasma inside the cusp region (Heikkila and Winningham, 1971). Reconnection between terrestrial magnetic field lines and the interplanetary magnetic field (IMF) is responsible for the penetration of solar wind plasma into the magnetosphere (e.g. Dungey, 1961; Dungey, 1963). Under southward IMF $B_{Z}$, IMF field lines may reconnect near the subsolar point and will subsequently convect poleward and antisunward, forming a broad cusp region. At mid-altitudes

Published by Copernicus GmbH on behalf of the European Geosciences Union. 


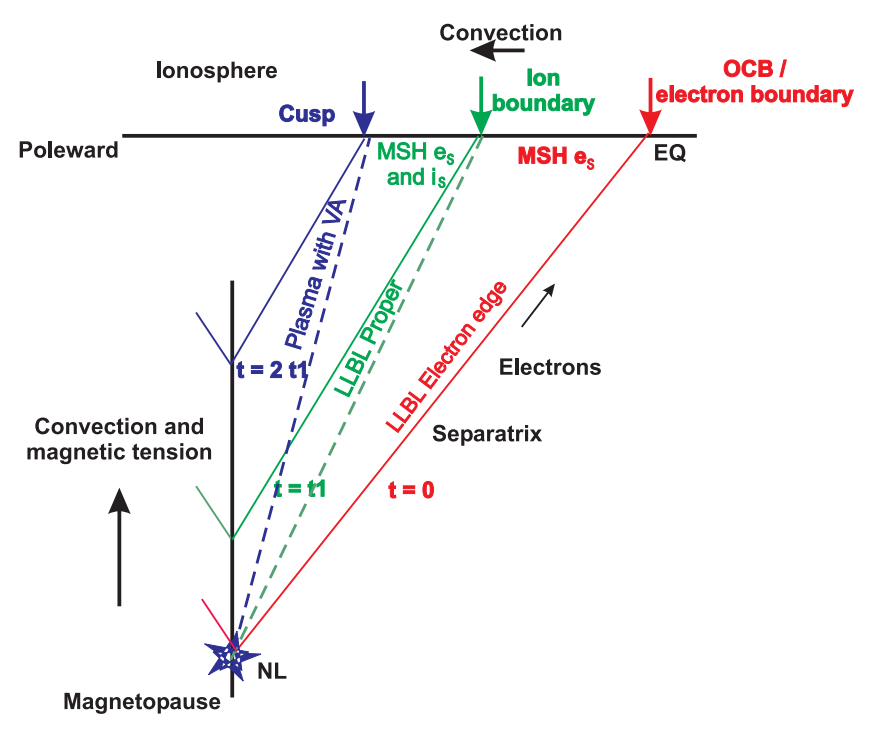

Fig. 1. Simplified schematic view of the evolution of magnetic field lines following reconnection, and the corresponding plasma regions at mid-altitudes or in the ionosphere. In this figure, all field lines have been straightened so that the magnetopause is represented by the vertical black line towards the left of the figure, and the ionosphere by the horizontal black line towards the top. The blue star shows location of the reconnection at the magnetopause. The red line represents a newly reconnected field line with time history since reconnection $t=0$. This line is the separatrix between closed and open field lines and also represents the electron equatorward boundary of the LLBL/cusp. The green line represents an older reconnected field line with time history since reconnection $t=t_{1}$ along which the most energetic magnetosheath ions have arrived at midaltitudes. The trajectory of these energetic ions is indicated by the dashed green line. This line represent ion equatorward boundary of the LLBL/cusp. Due to the magnetic tension, this line will convect away from the reconnection site, as indicated by the green solid line. The blue line represents an even older field line, with time history since reconnection $t=t_{2}$, along which the bulk plasma flow accelerated at the magnetopause up to the local magnetosheath Alfvén velocity will arrive at mid-altitudes. The trajectory of the plasma bulk flow is indicated by the dashed blue line. This line marks the equatorward boundary of the cusp proper. The region between the electron and ion boundaries of the LLBL/cusp is named the electron edge of the LLBL and contains only magnetosheath-like electrons but still magnetospheric ions. The region between the ion boundary of the LLBL/cusp and equatorward boundary of the cusp proper is the LLBL proper, containing both accelerated magnetosheath-like electrons and ions. The region poleward of the equatorward boundary of the cusp is the cusp proper.

(5-7 $R_{E}$ ), time-of-flight effects for particles injected by subsolar reconnection define the typical signatures of the plasma in the cusp (Smith and Lockwood, 1996). These include the energy-latitude dispersions and the low-energy cut-offs which are often identified in energy-time spectrograms of ion data recorded in the cusp.
At lower latitudes than the cusp proper, any low- or midaltitude satellite should cross two boundary layers with their own distinct plasma properties, but both resulting from dayside magnetic reconnection (Gosling et al., 1990; Lockwood, 1997). Figure 1 shows a simplified schematic view of the evolution of magnetic field lines following reconnection, and the corresponding plasma regions at mid-altitudes or in the ionosphere. In this figure, all field lines have been straightened so that the magnetopause is represented by the vertical black line towards the left of the figure, and the ionosphere by the horizontal black line towards the top. The most recently reconnected field line (represented by the red line in Fig. 1) corresponds to the separatrix, or boundary between closed terrestrial field lines and open, reconnected field lines which map into the magnetosheath. This "Open-Closed Boundary" (OCB) also maps back to the active neutral line (NL). Along this field line (or OCB) electrons of magnetosheath origin moving with very high velocity (Gosling et al., 1990) will arrive in the ionosphere almost immediately after the field lines become open. The arrival of these magnetosheath-like electrons is therefore a good marker of the OCB and the electron boundary of the Low-Latitude Boundary Layer (LLBL) (Lockwood, 1997; Onsager and Lockwood, 1997). However, due to the difference between typical ion and electron velocities, even the most energetic ions will not arrive at midaltitudes without a significant delay from the electron arrival (estimated to be up to $12 \mathrm{~min}$ by Topliss et al., 2001). Due to the poleward convection of the foot-points of reconnected field lines, this time delay translates into a significant spatial (latitudinal) separation of the location of the lowest-latitude part of the electron injections and the lowest-latitude ion injections. The part of the LLBL containing magnetosheathlike electrons but only the pre-existing magnetospheric-type ions is named the "electron edge" of the LLBL (Lockwood, 1997; Topliss et al., 2001; Sandholt et al., 2002). The arrival of energetic ions at low altitudes marks the ion boundary of the LLBL proper (Lockwood, 1997). These are more energetic and have lower fluxes than the ions observed at higher latitudes in the cusp proper (Newell and Meng, 1988). The latter region is formed at mid- and low-altitudes by the arrival of the bulk plasma injected from the magnetosheath and which moves down the convecting field lines with speeds comparable to the Alfvén velocity.

While statistical plasma properties of the LLBL and cusp regions have been extensively studied (e.g., Smith and Lockwood, 1996; Newell and Meng, 1988, 1992; Onsager et al., 1995; Lockwood and Smith, 1994; Lockwood et al., 1998; Aparicio et al., 1991; Newell et al., 1991a,b; Woch and Lundin, 1992, 1993; Zhou et al., 2000), the electron edge of the LLBL containing only magnetosheath-like electrons is a comparatively poorly studied region. However, Wing et al. (1996) and Wing et al. (2005) modelled the penetration of the magnetosheath electrons to low altitudes and compared results from these models with DMSP observations. It was shown that a parallel electric field between the magnetopause 
and low-altitudes must exist in order to adequately model the observed populations. This electric field acts to prevent the penetration of solar wind electrons along field lines ahead of ions, and thus conserves the quasi-neutrality of the plasma. However, the suprathermal "halo" part of the solar wind distribution will not be fully retarded by this parallel electric field and may thus penetrate to low altitudes ahead of the injected ions. These electrons should therefore be detected by low- and mid-altitude satellites on open field lines inside the LLBL, the mantle and the polar cap. To date there has been only a single statistical study reporting observations of the electron edge of the LLBL at the equatorward boundary of the cusp and containing the halo part of the solar wind population (Topliss et al., 2001). However, Topliss et al. found only 6 events with a clear electron edge out of a total of 200 cusp crossings made by the Polar spacecraft. The Cluster spacecraft cross the mid-altitude cusp region of the Northern hemisphere from the dayside to nightside and, as we report here, often detect such an electron edge of the LLBL. The aim of the present study is therefore to perform a statistical investigation of this part of the LLBL and to examine how the size of this region might depend on different external parameters.

The manuscript is organised as follows: Sect. 2 gives a brief description of the Cluster instruments used in this study; Sect. 3 presents an illustrative example of Cluster observations from a typical mid-altitude cusp crossing with a pronounced electron edge; Sect. 4 contains a description of the assembled data set and the methodology of the statistical study; Sect. 5 presents the results of this study while Sect. 6 contains a discussion of these results. Finally we present our conclusions in Sect. 7.

\section{Orbit and instrument description}

The Cluster orbit has a perigee of $\sim 4 R_{E}$ and an apogee of $\sim 19.7 R_{E}$, an inclination of $\sim 90^{\circ}$, and an orbital period of $\sim 57 \mathrm{~h}$. Such an polar orbit is very favourable for studies of the cusp region, particular the mid-altitude cusp. The observations reported here were acquired by the Plasma Electron and Current Experiment (PEACE) (Johnstone et al., 1997), and by the Hot Ion Analyser (HIA) and Composition and Distribution Function (CODIF) sensors, which are parts of the Cluster Ion Spectrometry (CIS) experiment (Rème et al., 2001). These instruments are mounted on each of the Cluster satellites. The CODIF sensor combines a top-hat analyser with an instantaneous $360^{\circ}$ field of view, with a time of flight section to measure the complete 3-D distribution functions of the major ion species, $\mathrm{H}^{+}, \mathrm{He}^{++}, \mathrm{He}^{+}, \mathrm{O}^{+}$. The sensor covers the energy range between 0.02 and $38 \mathrm{keV} / \mathrm{q}$ with a time resolution of $4 \mathrm{~s}$. Each PEACE package consists of two sensors, the HEEA (High Energy Electron Analyser) and the LEAA (Low Energy Electron Analyser), mounted on diametrically opposite sides of the spacecraft. They are designed to measure the three dimensional velocity distributions of electrons in the range of $0.6 \mathrm{eV}$ to $\sim 26 \mathrm{keV}$, with a time resolution of $4 \mathrm{~s}$. The spacecraft potential was measured by the Electric Fields and Waves (EFW) instrument (Gustafsson et al., 2001).

\section{Example of a mid-altitude cusp crossing with an elec- tron edge to the LLBL}

In this section we present an example of observation of a mid-altitude cusp crossing which exhibited an electron edge near the equatorward boundary of the LLBL/cusp. This crossing occurred on 10 September 2002, between 13:4514:10 UT. In order to provide the context for this event, solar wind parameters observed by ACE spacecraft during the period around the Cluster LLBL/cusp crossing are shown in Fig. 2. The top three panels show the X-, Y-, and Zcomponents of the interplanetary magnetic field in the GSM coordinate system. The bottom three panels show the $\mathrm{X}$ component of the solar wind velocity, the density and the dynamic plasma pressure. The dashed box marks the time of interest, which corresponds to the Cluster crossing of the northern hemisphere LLBL/cusp. The calculated time lag is 59 min. During Cluster LLBL/cusp crossing, the IMF $B_{\mathrm{Z}}$-component was stable and strong, $B_{\mathrm{Z}} \sim-5.5 \mathrm{nT}$. The IMF $B_{Y}$-component was quite small and varied in the limit $\pm 1.5 \mathrm{nT}$. The IMF $B_{X}$-component was negative, $-5.5 \mathrm{nT}$. Such IMF orientation is favourable for reconnection between terrestrial and IMF field lines at the dayside magnetopause. The velocity of the solar wind was quite stable, $V_{X} \sim 405 \mathrm{~km} \mathrm{~s}^{-1}$. There are no strong variations in the solar wind density, $N \sim 5 \mathrm{~cm}^{-3}$ and dynamic pressure, $P \sim 1.4 \mathrm{nPa}$.

We turn now to consider the Cluster data for this event, which are shown in Fig. 3. For simplicity, we show only data from Cluster spacecraft 1 (SC1). The three top panels present data from the PEACE instrument: these contain energy-time spectrograms for the differential energy flux of electrons in the anti-parallel (panel a), perpendicular (panel b) and parallel (panel c) directions. The black trace around 10-12 eV indicates the level of the spacecraft potential, as measured by the EFW instrument on SC1. Observation of electrons with energies lower than the spacecraft potential corresponds to the photoelectron population of spacecraft origin and should be disregarded. The next four panels show the CIS/CODIF ion data comprising: the energy-time spectrogram of protons $\mathrm{H}^{+}$(panel d), the energy-time spectrogram of oxygen $\mathrm{O}^{+}$ions (panel e) and the pitch-angle $\left(0^{\circ}-180^{\circ}\right)$-time spectrograms for low-energy $(20<\mathrm{E}<800 \mathrm{eV})$ and high-energy $(0.8<E<38 \mathrm{keV})$ protons (panels $\mathrm{f}$ and $\mathrm{g}$, respectively). The differential energy flux is represented by the colour bars on the right of each panel. The remaining five panels present the plasma parameters recorded during the period, where the black traces in each panel correspond to electron data and red traces (where appropriate) correspond to ion data. The 


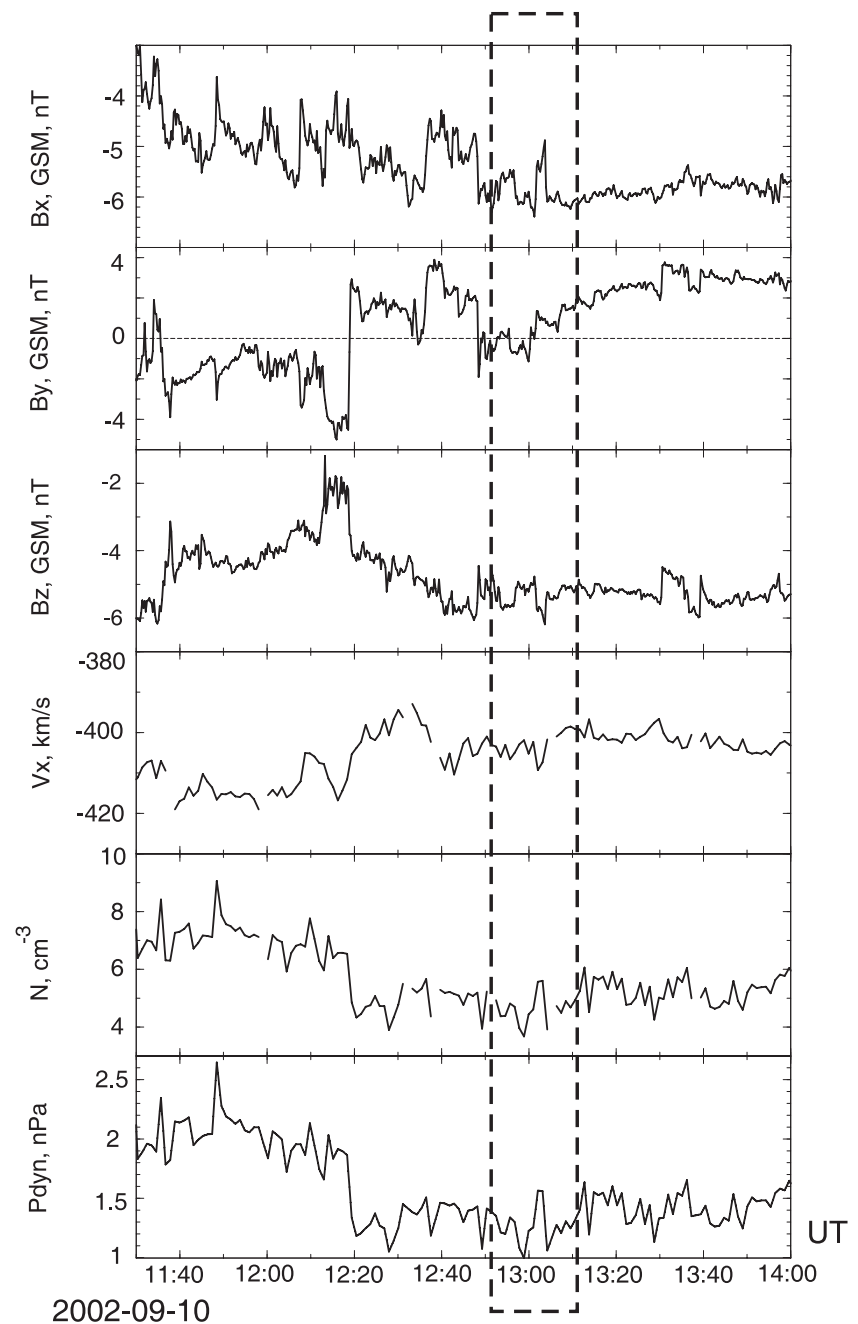

Fig. 2. Solar wind conditions for 10 September 2002. The top three panels show three components of the interplanetary magnetic field (IMF) in the geocentric solar magnetospheric (GSM) coordinate system. The bottom three panels show the X-component of the solar wind velocity (GSM), density and dynamic pressure of the solar wind. The dashed box marked the time of interest, when Cluster was in the northern LLBL/cusp. The appropriate time lag is $59 \mathrm{~min}$.

five panels show the plasma density (panel $\mathrm{h}$ ), the electron anisotropy, defined as $T_{\|} / T_{\perp}$, (panel i), the parallel to magnetic field plasma velocity (panel $\mathrm{j}$ ) and $\mathrm{X}$ - and $\mathrm{Y}$ - components of the plasma velocity perpendicular to magnetic field (panels $\mathrm{k}$ and $\mathrm{l}$ ). The three components of the plasma velocity are plotted in the same scale for inter-comparison. The ephemeris data for $\mathrm{SC} 1$ is presented under the plot, indicating X-, Y-, Z-components of position in the GSE coordinate system, magnetic local time (MLT) and invariant latitude (ILAT) of the observations. In the north cusp region, a positive parallel velocity corresponds to downgoing particles, the X-component of the perpendicular velocity repre- sents the plasma convection in the noon-midnight direction (positive towards Sun), and the Y-component of the perpendicular velocity shows plasma convection in the dusk-dawn direction (positive towards dusk). The plasma parameters presented are moments of the HIA 3-D ion distribution data and moments of the PEACE 3-D electron distribution data (the spacecraft was operating in burst mode during the time of interest, such that 3-D data was telemetered at a higher than usual rate from the spacecraft). The electron moments were averaged over $16 \mathrm{~s}$ in order to remove high-frequency variability and emphasize the main trends. There is some disagreement between the ion and electron moments: the plasma density obtained from the PEACE data is systematically a little higher than that from the HIA data. However, both density trends are in a good agreement and the velocity moments of the ion and electron distributions agree very well most of the time.

At the beginning of the time of interest, at 13:45 UT, SC1 was inside the dayside plasma sheet, characterised by the observation of a high energy, $1 \mathrm{keV}<E<30 \mathrm{keV}$, electron population which has fluxes perpendicular to the magnetic field rather higher than those parallel or anti-parallel, which is typical of trapped particles on closed field lines (Fig. 3, panels ac). On these field lines, there also exists a denser, low-energy electron population exhibiting peak fluxes along the field direction, which results in an electron parallel-to-perpendicular temperature anisotropy $\sim 1.2-1.3$ (panel i). High-energy plasma sheet ions are also evident in the CIS data (panel d). The density of the plasma is quite low in this region and varies in the range $0.5-2 \mathrm{~cm}^{-3}$ (panel h). The plasma convection and the parallel velocity of the plasma (bottom 3 panels) are also very low. At $\sim 13: 51$ UT (marked by the first dashed line) a dramatic change is observed in the electron distribution: the high energy population disappears and low-energy electrons, $(20<E<500 \mathrm{eV}$, consistent with a magnetosheath origin) become evident. This marks the electron boundary of the LLBL/cusp. However, note that magnetosheath-like ions do not arrive at SC1 until three minutes later, at 13:54 UT (marked by the second dashed line), as can most easily be seen in the pitch-angle spectrogram (panel g). This first appearance of magnetosheath-like $\mathrm{H}^{+}$ions with $0^{\circ}$ pitch-angles marks the ion boundary of the LLBL/cusp.

Between the electron and ion boundaries of the LLBL/cusp (i.e. between 13:51 and 13:54 UT), SC1 was inside the boundary layer which we identify as the electron edge of the LLBL. The invariant latitudes of the observed electron and ion boundaries are $74.77^{\circ}$ and $75.32^{\circ}$, respectively. Hence, the latitudinal size of the LLBL electron edge for this event, determined from the difference in the position of electron and ion injections, is $0.55^{\circ}$ ILAT. Note that this layer contains fairly isotropic electrons of magnetosheathlike energies. However, at low energies, $E=10-35 \mathrm{eV}$, the anti-parallel differential flux exceeds the parallel flux, suggesting the existence of an upgoing population of ionospheric origin. The level of anisotropy remains close to unity 


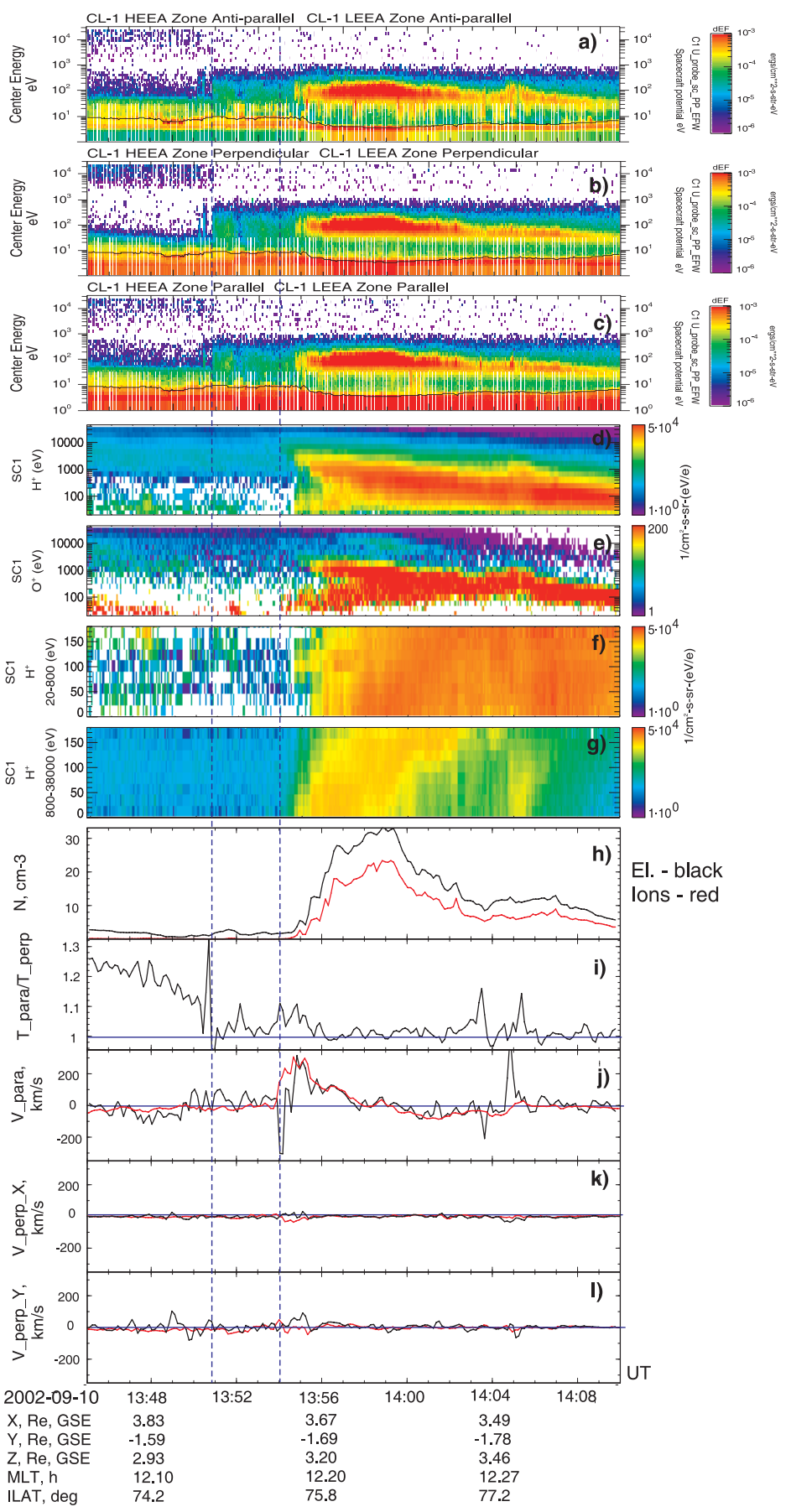

Fig. 3. PEACE and CIS data for the mid-altitude cusp crossing with the prominent electron edge of the LLBL on 10 September 2002, 13:4514:10 UT. From top to the bottom: electron energy-time spectrograms in the antiparallel (panel a), perpendicular (panel b) and parallel (panel c) directions; the energy-time spectrogram of protons $\mathrm{H}^{+}$(panel d); the energy-time spectrogram of oxygen $\mathrm{O}^{+}$ions (panel e); the pitch-angle $\left(0^{\circ}-180^{\circ}\right)$-time spectrograms for low-energy $(20<E<800 \mathrm{eV})$ and high-energy $(0.8<E<38 \mathrm{keV})$ protons (panels $\mathbf{f}$ and $\mathbf{g}$, respectively); the electron (black trace) and the ion (red trace) density (panel $\mathbf{h}$ ); the electron anisotropy, defined as $T_{\|} / T_{\perp}$, (panel $\mathbf{i}$ ); the parallel velocity of plasma (panel $\mathbf{j}$ ) and $\mathrm{X}$ - and Y-components of the perpendicular plasma velocity (panels $\mathbf{k}$ and $\mathbf{l}$ ). Black traces correspond to electron data and red traces correspond to ion data, and plasma velocities are shown in the same scale for inter-comparison. The ephemeris data for SC1 are presented under the plot: X-, Y-, Z-components of position in the GSE coordinate system, magnetic local time (MLT) and invariant latitude (ILAT) of observation. 
apart from a short time interval around 13:52 UT when a dense low-energy $(10<E<40 \mathrm{eV})$ upgoing electron population was detected. The density of electrons in this region is $3-5 \mathrm{~cm}^{-3}$. Note also, however, that this boundary layer still contains the high-energy, trapped protons of plasma sheet origin whose characteristics are largely unaltered from those observed prior to 13:51 UT. There is no significant plasma convection inside this region, the $\mathrm{X}$ - and $\mathrm{Y}$ - components of perpendicular velocity were close to $0 \mathrm{~km} \mathrm{~s}^{-1}$. The electron parallel velocity inside this region was slightly positive, suggesting perhaps that the flux of downgoing electrons is a little higher than that of the upgoing population. The average ion parallel velocity was $\sim 0 \mathrm{~km} \mathrm{~s}^{-1}$. The energy range of the observed electrons suggests that this boundary layer is on open field lines, which must have been recently reconnected somewhere on the dayside magnetopause. However, despite the appearance of the magnetosheath-like electrons, the convection observed at mid-altitudes is rather small inside this boundary layer. Consequently we conclude that these electrons do not carry sufficient current, and therefore do not yet have sufficient dynamic influence to drive the poleward convection of the footpoints of the reconnected field lines.

After 13:54 UT (the second dashed line at Fig. 3), SC1 crossed a small LLBL proper (13:54:00-13:55:30 UT). Inside this region, in addition to low-density magnetosheathlike electrons, $E=10-300 \mathrm{eV}$, low-density accelerated magnetosheath ions, $E=1-10 \mathrm{keV}$, are also observed. This highenergy ion population smoothly evolves with time into the cusp indicating the continuous time-of-flight effect. Inside the LLBL proper, the plasma density is still rather small but antisunward/poleward $\left(0<V_{\perp X}<50 \mathrm{~km} \mathrm{~s}^{-1}\right)$ convection is observed together with strong plasma injections (parallel velocity, $V_{\|}$, increases up to $350 \mathrm{~km} \mathrm{~s}^{-1}$ ). The electron anisotropy is relatively high, $\sim 1.2$, which is typical for the LLBL region (Woch and Lundin, 1993). In correlation with this electron anisotropy enhancement, the local heating of both low energy, $E=20-100 \mathrm{eV}$, ionospheric protons (with $90-100^{\circ}$ pitch-angles in panel f) and $\mathrm{O}^{+}$ions (oxygen pitchangles are not shown) become evident.

At 13:55:30 UT SC1 enters the cusp proper, which is characterised by the observation of high fluxes of dense magnetosheath-like plasma showing an energy-latitude dispersion and a low-energy cut-off in the ion fluxes (e.g. Smith and Lockwood, 1996). Inside this region, between 13:55:3014:00 UT, the plasma density increases to a maximum of $\sim 20-30 \mathrm{~cm}^{-3}$, and the electron population becomes quite isotropic, $\mathrm{T}_{\|} / \mathrm{T}_{\perp} \sim 1$, while the ion pitch-angle distributions show dispersions consistent with the time-of-flight effect. Inside the cusp, the convection velocity was very low. There is still a net downgoing flow of plasma inside the cusp, but the bulk flow velocity decreases with latitude. At $\sim 14: 00$ UT, $\mathrm{SC} 1$ enters the mantle region, which is characterized by the domination of upgoing mirrored population of ions $\left(V_{\|}<0\right)$. Inside the mantle the density of the plasma slowly decreases and the convection velocity is close to zero. At $\sim 14: 04$ UT the ion pitch-angle spectrogram suggests the occurrence of a new pulse of plasma injections, but this possibility is outside the scope of this paper.

\section{Statistical data set and methodology}

\subsection{Data set}

Electron edges of the LLBL, characteristics of which are similar to those described in the previous section, are frequently observed by the Cluster spacecraft near the equatorward boundary of the LLBL/cusp. A systematic statistical study of the position and size of this boundary layer, as observed at mid-altitudes, and their dependence on different external parameters, has been performed and results are reported here. In this statistical study, we have used 3 years of data from Northern hemisphere mid-altitude cusp crossings, occurring in the months July-October of years 2001-2003. For each event the electron boundary of the LLBL/cusp is identified in energy-time spectrograms by the simultaneous disappearance of the high-energy, trapped magnetospheric electron population and the arrival of low-energy, low-density magnetosheath-like electrons. We exclude events which show a smooth transition between these two regions, for example when there are still significant fluxes of high-energy electrons in the cusp, or when the LLBL is obviously on closed field lines. The ion boundary of the LLBL is then identified in the pitch-angle spectrograms of the protons by the arrival of magnetosheath-like ions with energies less than 4-6 keV and with pitch-angles of $\sim 0^{\circ}$. Again, we selected events only with clear ion boundaries.

To estimate the relevant solar wind and IMF conditions, data from ACE, located near the L1 Lagrangian point, have been used. The time lag associated with solar wind convection between our Cluster observations and the ACE observations was calculated based on the X-component of the solar wind velocity, $V_{\mathrm{Xsw}}$ in GSE coordinate system (measured with a resolution of $64 \mathrm{~s}$ ) and the position of the ACE along Sun-Earth line (GSE X-axis). The time lag was defined as $\Delta t=X / V_{\mathrm{Xsw}}$, where $X$ is the distance from ACE to the centre of the Earth in the GSE X-direction. This method was also applied in the statistical study by Stubbs et al. (2004). In the calculating the time to the centre of the Earth, we assume that the time taken for the shocked solar wind plasma to travel from the bowshock to the sub-solar magnetopause through the magnetosheath is roughly equal to time needed for the normal solar wind to travel from the stand-off bowshock distance to the Earth (approximately 2-3 min). The appropriate solar wind velocity was calculated as follows: if $t_{0}$ is a time when Cluster crosses the electron boundary of the LLBL/cusp, for a first attempt we estimated the average solar wind velocity measured by ACE over period 40-70 min before $t_{0}$, calculating $V_{\mathrm{Xsw} 0}$. If the velocity of the solar wind is quite stable during this averaged $30 \mathrm{~min}$ period, we use 
the calculated solar wind velocity, $V_{\mathrm{Xsw}}=V_{\mathrm{Xsw} 0}$, to derive the time lag between Cluster and ACE. If solar wind velocity was highly variable during this period, we estimate the time lag based on average velocity, $\Delta t_{0}=X / V_{\mathrm{Xsw} 0}$, and then estimate the solar wind velocity, $V_{\mathrm{Xsw} 1}$, in a 10 -min interval around time $t_{0}-\Delta t_{0}$. We then used this solar wind velocity, $V_{X s w}=V_{X s w 1}$, to estimate the final, more accurate, time lag between Cluster and ACE.

Since we concentrate here on the boundary layer located near the equatorward edge of the cusp, which is formed due to reconnection at the dayside magnetopause, events with long-lasting northward IMF have also been excluded from the study. For this IMF orientation reconnection on the lobe magnetopause, poleward of the cusp is more likely (Dungey, 1963; Crooker, 1979) and thus a similar boundary layer should form near the poleward boundary of the cusp. For events with a highly variable IMF $B_{Z}$-component, the energy-time and pitch-angle spectrograms have been examined in closer detail. We include events under temporarily northward IMF if there are combined observations of "normal" energy-latitude dispersion, low-energy ion cut-offs and arrival of the protons with $0^{\circ}$ pitch-angles at the equatorward boundary of the cusp. Each of these observations are considered signatures of reconnection at the dayside magnetopause (e.g. Smith and Lockwood, 1996).

In total we have analysed 327 mid-altitude LLBL/cusp crossings by Cluster. Out of this total, we have identified 129 events ( $\sim 40 \%$ of all crossings) with clear electron and ion equatorward boundaries of the LLBL/cusp and with cusp plasma signatures corresponding to the dayside magnetopause reconnection. For these 129 events we have estimated the size of the electron edge of the LLBL using the "one-spacecraft" or "multi-spacecraft" methods discussed in the following sections.

\section{2 "One-spacecraft" method}

The orbital dynamics of the Cluster spacecraft often lead to significant temporal separations between passages of the individual spacecraft through the mid-altitude cusp. For example, during seasons 2001 and 2002, SC3 trailed the other spacecraft by several tens of minutes, such that its observations of the cusp should be considered an observation by a single spacecraft only. In such cases, the size of the electron edge of the LLBL is defined simply as the difference between invariant latitudes of the electron and ion boundaries observed by the spacecraft, i.e., $\operatorname{Size}=I L A T_{\mathrm{ions}}-I L A T_{\mathrm{el}}$. The time difference between observations of the electron and ion boundaries by one spacecraft in our dataset varied between 0 and $5 \mathrm{~min}$. Such "one-spacecraft" estimations will be accurate providing the boundaries do not move much between observations of these two boundaries by the spacecraft. However, the boundaries in the LLBL/cusp can be highly dynamic and respond quickly to any changes of the reconnection geometry and/or the IMF/solar wind conditions. For exam- ple, for stable southward IMF, magnetopause erosion is frequently observed (e.g. Farrugia et al., 2001), meaning that the OCB and the electron boundary are constantly drifting equatorward (Yeoman et al., 2002). The rapid equatorward motion of the cusp/cleft aurora observed during period of stable southward IMF also confirms this (e.g. Karlson et al., 1996). The small statistical shift of the cusp position equatorward has also been detected for increased $B_{Y}$-components of IMF, suggesting the occurence of dayside magnetopause erosion during events with strong dawn-dusk components of the IMF (Wing et al., 2001). Moreover, variations in the IMF $B_{Y}$-component could change the position in MLT of the cusp proper and that of the surrounding boundary layers (Smith and Lockwood, 1996; Aparicio et al., 1991; Zhou et al., 2000; Russell, 2000; Merka et al., 2002; Karlson et al., 1996; Moen et al., 1999). Changes in the IMF $B_{Z}$-component may also shift the latitudinal position (Woch and Lundin, 1992; Zhou et al., 2000; Russell, 2000). In these situations, the one-spacecraft estimation of this boundary size may contain a significant error, as the ion and electron boundaries may have moved between observations by one spacecraft at different times. Note that the critical dependency is on the time between the detection of the electron and ion boundaries: if that time is small, the uncertainty of the estimation will be small. However, the accuracy of size estimation decreases with increasing time difference between observations.

\subsection{Multi-spacecraft method}

With Cluster we have the advantage of using multi-spacecraft observations to estimate the motion of the OCB and electron/ion boundaries with time. Figure 4 illustrates this concept and our methodology for making a more accurate measurement of the width of the electron edge. This figure is a plot of invariant latitude versus time, on which we plot the positions of the boundaries observed by each spacecraft. If all four Cluster spacecraft enter the cusp and the surrounding boundary layers, we will have 4 measurements of both the magnetic local time (MLT) and invariant latitude (ILAT) of the observed electron boundary of the LLBL/cusp at four different times. By way of an illustrative example, we mark 4 such points as $1 e, 2 e, 3 e$, and $4 e$ in Fig. 4 . We also will have 3 measurements of the MLT and ILAT of the ion boundary of the LLBL/cusp (since CIS is unfortunately not working on SC2) at three different times. Such points are also marked as $l i, 3 i$, and $4 i$ in Fig. 4. Based on 4 measurements of the invariant latitude of the electron boundary by different spacecraft we are able to reconstruct an estimation of the electron boundary motion (ILAT versus time) using a linear least squares fit (as illustrated by the red line on Fig. 4). Using the reconstructed electron boundary, we are able to estimate the invariant latitudes of this boundary at the times when the Cluster satellites detect the ion boundaries. These reconstructed invariant latitudes are marked as le new, 3e new, and $4 e_{-}$new in Fig. 4. Therefore, in the "multi-spacecraft" 


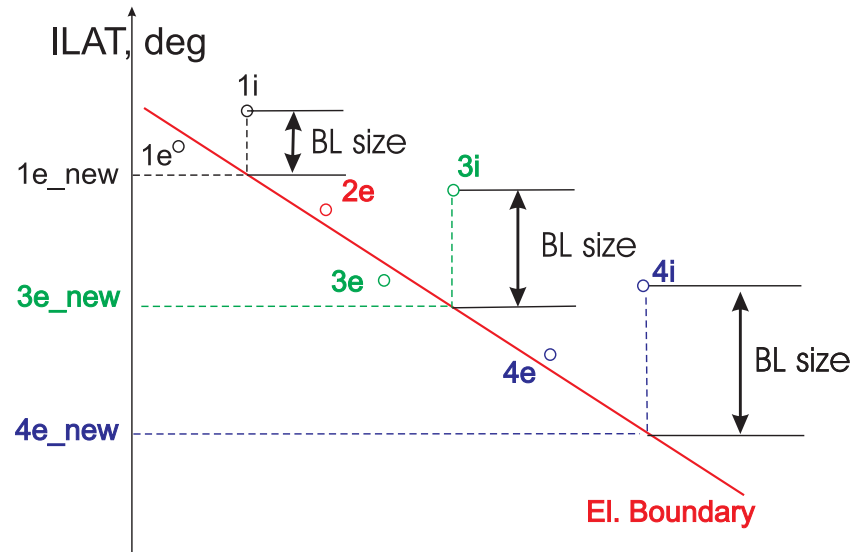

Time

Fig. 4. Illustration of the multi-spacecraft method used in this paper, showing a plot of invariant latitude versus time. Points marked as $1 e, 2 e, 3 e$ and $4 e$ indicate 4 measurements of invariant latitude of the observed electron boundary of the LLBL/cusp at different times made by SC $1,2,3$ and 4 , correspondingly. Points $1 i, 3 i$ and $4 i$ indicate 3 measurements of invariant latitude of the observed ion boundary of the LLBL/cusp at different times made by SC1, 3 and 4 correspondingly. The red line represents the position of the electron boundary as a function of time reconstructed from the linear least squares fit to the four measurements $1 e, 2 e, 3 e$, and $4 e$. Points marked as $1 e \_n e w, 3 e \_n e w$ and $4 e \_n e w$ represent reconstructed invariant latitudes of the electron boundary the times when the Cluster satellites detect the ion boundaries $1 i, 3 i$ and $4 i$.

method, the size of the electron edge of the LLBL is calculated as the difference between the invariant latitude of the observed ion boundary and the invariant latitude of the reconstructed electron boundary at the same time: Size $=I L A T_{i o n s}$ - ILAT el_new. For the example presented in Fig. 4, it is evident that the size of the electron edge estimated from the multi-spacecraft method will be larger than that estimated from the one-spacecraft method. This will typically be the case for events during which magnetopause erosion is occurring.The multi-spacecraft method can be used with high accuracy under two assumptions: (1) the OCB and electron boundary are aligned along invariant latitude; (2) the motion of the OCB and electron boundary between observations by different spacecraft is linear. In the former case, we note that the spacecraft separation in MLT in the midaltitude cusp varied between $0.1 \mathrm{~h}-0.5 \mathrm{~h}$ MLT, so we assume that differences in observations by different spacecraft due to MLT separation can be neglected. The multi-spacecraft method was applied based on 4-point electron measurements during the 2003 season and on 3-point measurements of the electron boundary for events from 2001 and 2002, as SC3 was very far away from the other three spacecraft (typically crossing the cusp after a time lag of $30-40 \mathrm{~min}$, as noted above). We do not believe that the assumption of linear mo- tion of the OCB can often realistically be applied over such a long time interval. The time of crossings of the electron boundary by the closely separated spacecraft varied between $20 \mathrm{~s}$ and $\sim 10 \mathrm{~min}$, so the accuracy of the electron boundary motion reconstruction could vary between events. However, we assume that the error of the size estimation is not higher than $0.1^{\circ}$ ILAT. For the example discussed above (10 September 2002) the SC crossed $1^{\circ}$ ILAT in $5 \mathrm{~min}$, corresponding to $30 \mathrm{~s}$ for $0.1^{\circ}$ ILAT. The PEACE and CIS data have a time resolution of $4 \mathrm{~s}$, while the orbits of the SC are reconstructed from orbit files with a 5-min resolution. Taking these points together with the linear fits to the observed data, the error of $0.1^{\circ}$ ILAT seems reasonable. However, if the multi-spacecraft method returned an unrealistically large size estimation which was very different from the one-spacecraft method applied to each spacecraft, we used size estimated from the latter method in the statistical study (relevant to 10 events). In this study, we were able to perform the multi-spacecraft estimation of the electron edge size for 83 events, and we have used the "one-spacecraft" estimation of the LLBL electron edge size for the rest of the events (46 events). We determined then how the size of the LLBL electron edge depends on the IMF components and magnitude; on the solar wind dynamic pressure, velocity and density; on the invariant latitude, magnetic local time and level of the geomagnetic activity $K_{\mathrm{p}}$.

\subsection{Data set}

Figure 5 describes the breakdown of the overall data set in the form of histograms, showing how many events were observed under different conditions. The plots each show the total number of events in each bin (black lines) and the number of those events for which the multi-spacecraft technique for the estimation of the LLBL electron edge size could be employed (red lines). The top row of plots indicates where the electron boundary of the LLBL/cusp has been observed. The majority of events were observed at 73-80 ILAT and 10:00-15:00 MLT. The next 2 rows indicate the range of IMF conditions $\left(B_{Y}, B_{Z}\right.$, clock angle and $B_{X}$ ) prevailing at the time of observation. The $B_{Y}$ component of the IMF mainly is in the range $\pm 8 \mathrm{nT}$, with most events with $B_{Y} \sim \pm 2-3 \mathrm{nT}$, and the IMF $B_{Z}$-component mainly lies in the range $\pm 5 \mathrm{nT}$, with most events with $B_{Z} \sim-$ $3 \mathrm{nT}$. The IMF clock angle, $\Phi_{C A}=\tan ^{-1}\left(B_{Y} / B_{Z}\right)$ for the observed events varies between $\pm 10^{\circ}$ and $\pm 180^{\circ}$, with most events having a clock angle of $\pm\left(60^{\circ}-110^{\circ}\right)$. For the majority of events the IMF $B_{X}$-component lies in the range $\pm 6 \mathrm{nT}$, with most events at $-3 \mathrm{nT}$. The fourth row of plots shows the solar wind density and velocity prevailing at the time of the events. For the majority of the observations, the solar wind conditions were near average, with density in the range $N_{\mathrm{sw}}=0.5-10 \mathrm{~cm}^{-3}$ with a peak at $N_{\mathrm{sw}}=2-3 \mathrm{~cm}^{-3}$; and velocity $V_{\mathrm{sw}}=300-750 \mathrm{~km} \mathrm{~s}^{-1}$ with a peak at $V_{\mathrm{sw}}=400-500 \mathrm{~km} \mathrm{~s}^{-1}$. The corresponding solar wind 

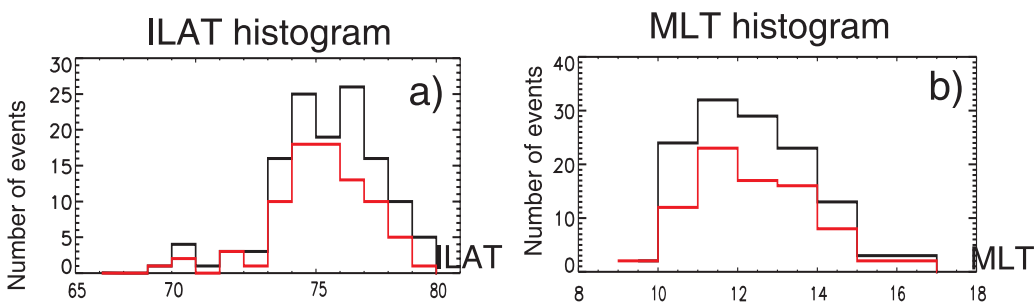

By histogram
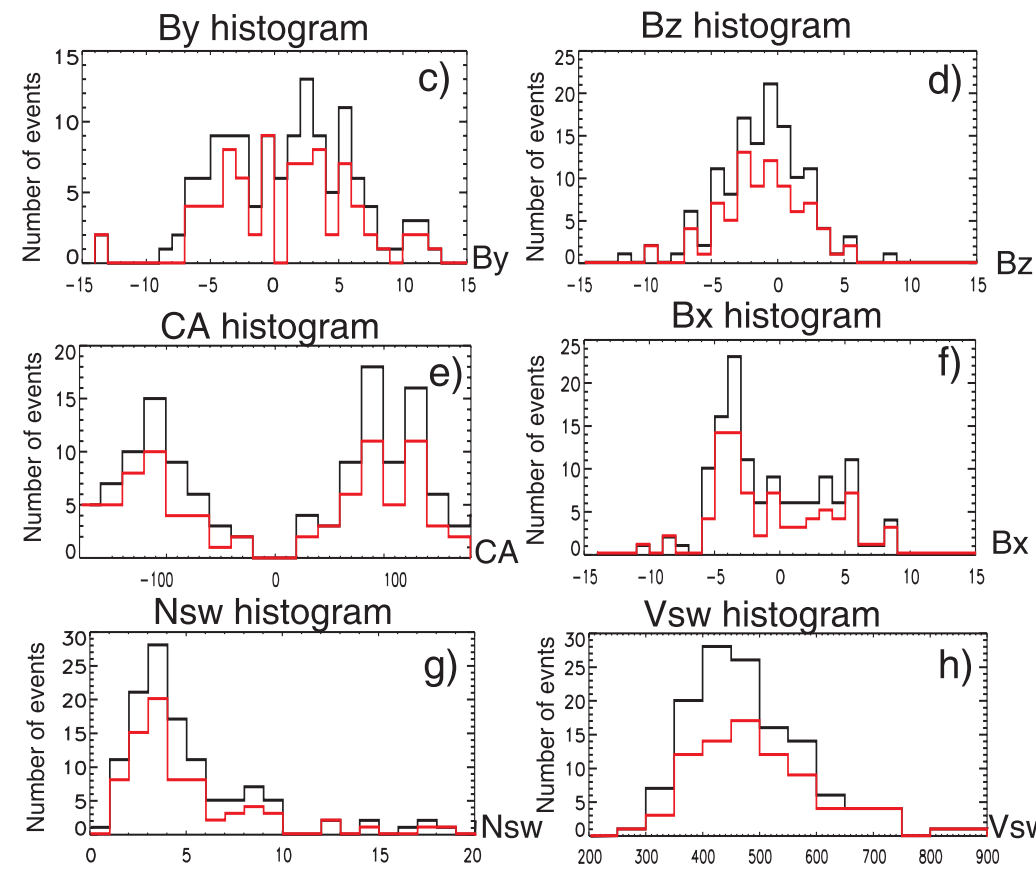

Vsw histogram
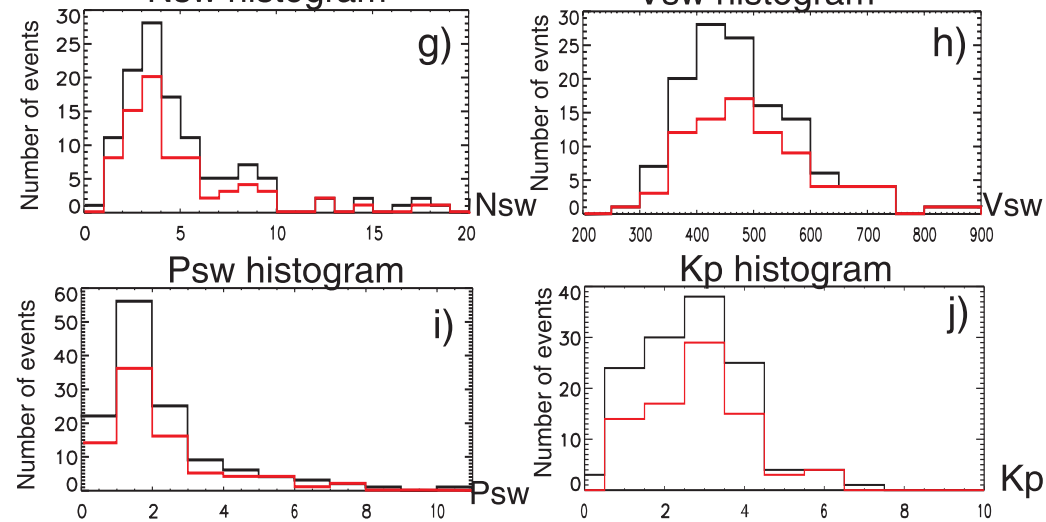

Fig. 5. Properties of the available data set in the form of histograms showing how many events were observed under different conditions: the invariant latitude of observations (panel a), the magnetic local time of observations (panel b), the IMF $B_{\mathrm{Y}}$-component and $B_{\mathrm{Z}}$-component (panels $\mathbf{c}$ and $\mathbf{d}$, correspondingly), the IMF clock-angle (panel e), the IMF $B_{X}$-component (panel f), the solar wind density (panel $\mathbf{g}$ ), the solar wind velocity (panel $\mathbf{h}$ ), the solar wind dynamic pressure (panel i), and index of geomagnetic activity $K_{\mathrm{p}}$ (panel $\mathbf{j}$ ). The GSM coordinate system has been used. The plots each show the total number of events in each bin (black lines) and the number of those events for which the multi-spacecraft technique for the estimation of the LLBL electron edge size could be employed (red lines).

dynamic pressure, shown in the bottom left panel, $P_{\mathrm{sw}}=0.2$ $4 \mathrm{nPa}$ with a peak at $P_{\mathrm{sw}}=1-2 \mathrm{nPa}$. Events were observed mainly during periods of low or medium geomagnetic activity, $K_{\mathrm{p}}=0-5$ (bottom right panel).

4.5 Invariant latitude of the equatorward electron boundary of the LLBL as function of external parameters

Figure 6 shows how invariant latitude, ILAT, of the electron boundary of the LLBL/cusp depends on IMF $B_{Z}$-component (left panel) and solar wind dynamic pressure (right panel).
The horizontal red bars on the left panel show the median values of the ILAT for every $2 \mathrm{nT}$ of IMF $B_{\mathrm{Z}}$-component. Median values, as well as the scatter plot, indicate that for IMF $B_{\mathrm{Z}}<0$, when IMF is more southward, the electron boundary of the LLBL/cusp moves equatorward. However, when IMF $B_{\mathrm{Z}}>0$, the invariant latitude of the LLBL/cusp equatorward electron boundary is fairly steady. On the right panel of Fig. 6, the horizontal red bars show the median invariant latitudes every $1 \mathrm{nPa}$ of the solar wind dynamic pressure. The trend in the median values suggests that the 

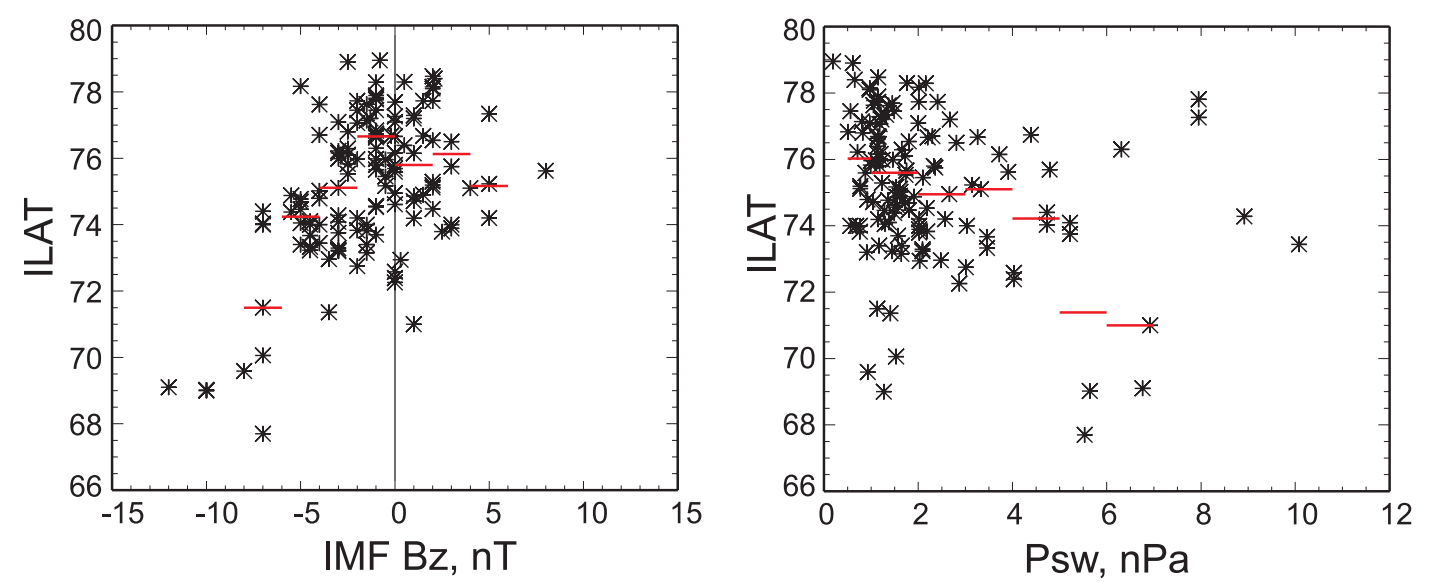

Fig. 6. Correlation of the invariant latitude ILAT of the electron boundary of the LLBL/cusp and external parameters: IMF $B_{Z}$-component (left panel) and solar wind dynamic pressure (right panel). The horizontal red bars in the figures show the median values of the ILAT in each bin.

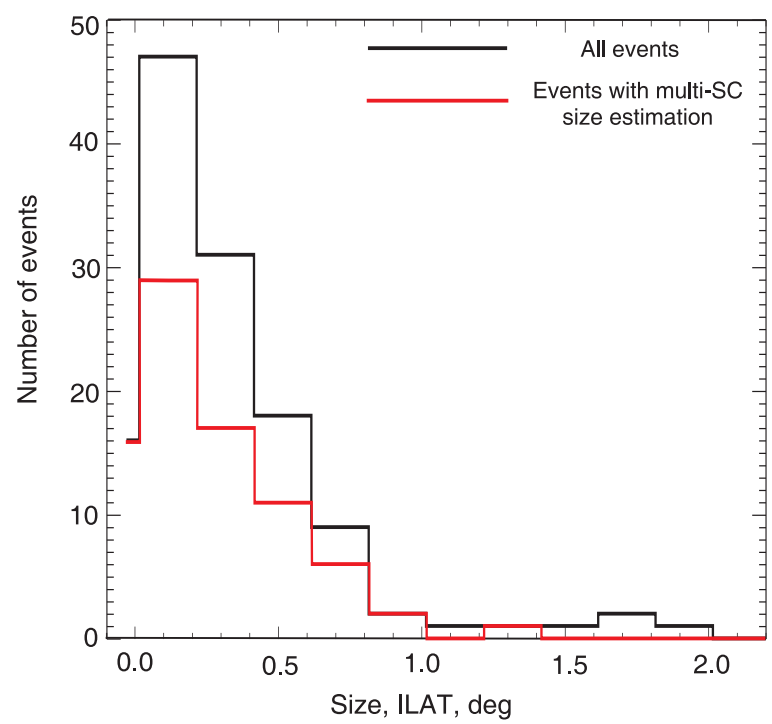

Fig. 7. Histograms showing the distribution of sizes of the electron edge of the LLBL, for all events (black line) and for events in which we obtained a multi-spacecraft estimation of the size (red line).

invariant latitude of the electron equatorward boundary of the LLBL/cusp shifts equatorward for increased solar wind dynamic pressure. We would like to notice that despite of median values show some dependency, there are a lot of scattering in the data points around these median values.

\subsection{Size of the electron edge of the LLBL}

Figure 7 presents histograms showing the distribution of sizes of the electron edge, for all events (black line) and for events in which we obtained a multi-spacecraft estimation of the size (red line). Note that only 16 events ( $13 \%$ of all events with clear electron and ion boundaries) do not show a distinct electron edge, i.e. for these events the ion and electron boundaries were observed simultaneously. The size of the electron edge of the LLBL varies between $0^{\circ}$ and $2^{\circ}$ ILAT, with a mean value of $0.3^{\circ}$ ILAT and a median value of $0.2^{\circ}$ ILAT. The median value is more meaningful as it excludes the influence of a few extreme points. There are seven events where the electron edge size is greater than $1^{\circ}$ ILAT. However, five of these relied on an estimation of size from the one spacecraft method. Hence, in these cases the accuracy could be low, as discussed above. The majority of events have size $0.05-0.2^{\circ}$ ILAT with monotonically decreasing number of events with increasing size.

\subsection{Dependence of the electron edge size on different pa- rameters}

Figure 8 shows how the size of the electron edge of the LLBL depends on the magnitude of the IMF (panel a), the IMF $B_{X^{-}}$ component (panel b, GSM coordinate system was used), the invariant latitude of observations ILAT (panel c), the geomagnetic index $K_{\mathrm{p}}$ (panel d), the dynamical pressure of the solar wind (panel e), the Magnetic Local Time (MLT) of the observation (panel f), the solar wind density (panel g), the $B_{\mathrm{Y}^{-}}$and $B_{\mathrm{Z}}$-components of IMF (panels h and i, GSM coordinate system was used) and the solar wind velocity (panel $\mathrm{j}$ ). The panels are listed in order of the level of dependency of the electron edge size on external parameters: i.e. panel (a) shows the best dependency and panel (j) shows the worst dependency. For these calculations, we have combined events with the size estimated from either multi-spacecraft (if available) or one-spacecraft methods to increase statistics. In each panel, the red points represent events where the size was estimated from the multi-spacecraft method, while the black 

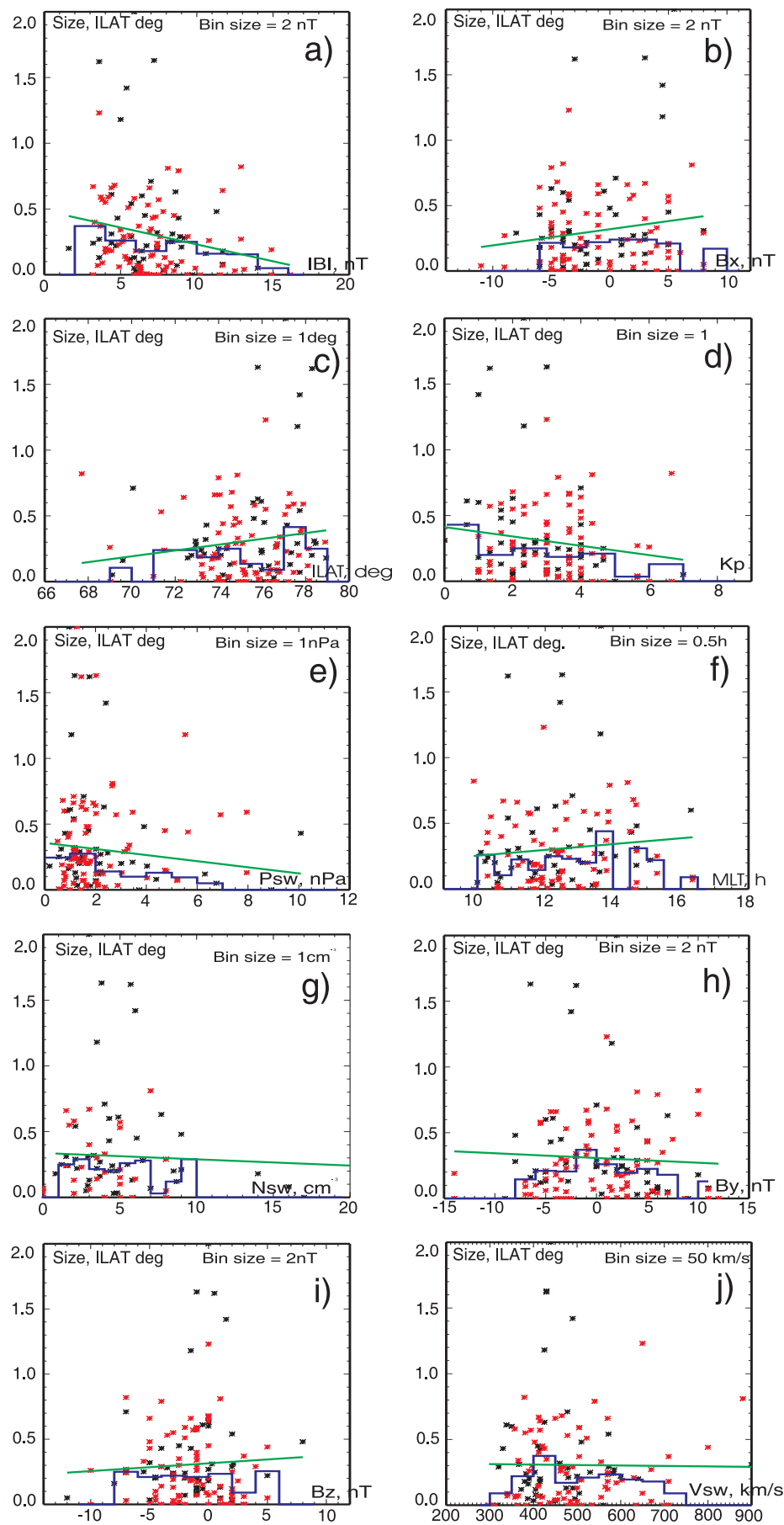

Fig. 8. Dependencies of the size of the electron edge of the LLBL on external parameters: the magnitude of the IMF (panel a), the IMF $B_{\mathrm{X}}$-component (panel $\mathbf{b}$ ), the invariant latitude of observations ILAT (panel $\mathbf{c}$ ), the geomagnetic index $K_{\mathrm{p}}$ (panel $\mathbf{d}$ ), the dynamic pressure of the solar wind (panel e), the Magnetic Local Time (MLT) of the observation (panel f), the solar wind density (panel $\mathbf{g}$ ), the $B_{\mathrm{Y}^{-}}$and $B_{\mathrm{Z}^{-}}$ components of IMF (panels $\mathbf{h}$ and $\mathbf{i}$ ) and the solar wind velocity (panel $\mathbf{j}$ ). The GSM coordinate system has been used. The panels are listed in order of the strength of the dependency of the electron edge size on the external parameter: i.e. panel (a) shows the best dependency and panel (j) shows the worst dependency. In each panel, the red points represent events where the size was estimated from the multi-spacecraft method, while the black points represent events where the size was estimated from the one-spacecraft method. The green line shows a linear least squares fit to these data, while the blue line shows the median value of the size in each bin, for cases where there are more than 3 points in a bin. 
Table 1. Correlation coefficients and statistical significances between the electron edge size and external parameters and indication of the presence of a trend in the median values of the size.

\begin{tabular}{lccc}
\hline Parameter & $C C$ & $S S$ & Median value trend \\
\hline IMF mag $(B)$ & -0.20 & $98 \%$ & Yes \\
IMF $B_{\mathrm{X}}$ & 0.15 & $91 \%$ & No \\
ILAT & 0.14 & $89 \%$ & Yes \\
$K_{\mathrm{p}}$ & -0.14 & $89 \%$ & Yes \\
$P_{\text {Sw }}$ & -0.12 & $82 \%$ & Yes \\
MLT & 0.09 & $69 \%$ & No \\
$N_{\text {sw }}$ & -0.09 & $69 \%$ & No \\
IMF $B_{\mathrm{Y}}$ & -0.05 & $43 \%$ & No \\
IMF $B_{\mathrm{Z}}$ & 0.05 & $43 \%$ & No \\
$V_{\text {sw }}$ & -0.01 & $9 \%$ & No \\
\hline
\end{tabular}

Table 2. Correlation coefficients, statistical significances and the presence of obvious trends in the median values between electron edge size and MLT shift from the local noon, and between electron edge size and absolute values of the IMF components.

\begin{tabular}{lccc}
\hline Parameter & $C C$ & $S S$ & Median value trend \\
\hline MLT shift from local noon & 0.04 & $35 \%$ & Yes \\
IMF mag $\left(B_{\mathrm{Y}}\right)$ & -0.185 & $96.3 \%$ & Yes \\
IMF $\operatorname{mag}\left(B_{\mathrm{Z}}\right)$ & -0.166 & $93.8 \%$ & Yes \\
IMF $\operatorname{mag}\left(B_{\mathrm{X}}\right)$ & -0.02 & $12 \%$ & No \\
\hline
\end{tabular}

points represent events where the size was estimated from the one-spacecraft method. The green line shows a linear least squares fit to these data, while the blue line shows the median value of the size in each bin, for cases where there are more than 3 points in a bin. We have estimated the Pearson correlation coefficient $C C$ (Edwards, 1976) and statistical significance of the result SS. The statistical significance was calculated based on Student's t-test (Devore, 1995). Following other statistical studies (McCall, 1990), we consider that correlation with $S S>95 \%$ is a statistically significant result. This means that the probability of two random sets of data of the same size as our dataset showing the same correlation coefficient as we had in a statistical study is only 5 percent. Table 1 summarizes our results in support of Fig. 8 . It shows the external parameter tested for correlation with the electron edge size, the Pearson correlation coefficient, the statistical significance of the correlation and whether we can see clear trend in the median values or not. The results are sorted according to the correlation coefficient.

Panel (a) of Fig. 8 shows the best dependency between the size of the electron edge and an external parameter obtained in our study. An anti-correlation between the electron edge size and the IMF magnitude is evident. The correlation coefficient between these values was $C C=-0.20$ (Ta- ble 1). This means that $20 \%$ of variations in the electron edge size correspond to variations in the IMF magnitude. This result has high statistical significance, $S S=98 \%$. Similar anticorrelation is also seen in the median values. Panel (b) of Fig. 8 shows how the electron edge size depends on the IMF $B_{\mathrm{X}}$-component. Formally this is our second most significant result, with the correlation coefficient $C C=0.15$ and a statistical significance of the result $S S=91 \%$. However, the $S S$ of result is already lower than traditional level of $95 \%$. Moreover, we did not see an obvious dependency in the median in the bin values. Panels (c-e) of Fig. 8 show examples of noticeable trends in the median values between the size of the electron edge and external parameters which could be suggestive of some dependencies. However correlations between the electron edge size and the invariant latitude, the $K_{\mathrm{p}}$ index and the solar wind dynamic pressure are smaller ( $C C=-0.14 ;-0.14 ; 0.12$, correspondingly) as well as statistical significance of the results ( $S S=89 \% ; 89 \% ; 82 \%$, correspondingly). These results in general should not thus be considered as statistically significant. We still consider trends in the median values as an important result, as (1) the Pearson correlation corresponds to the simplest situation of a linear least squares fit and for our data we probably need to use more sophisticated analysis (such as polynomial or exponential fits, for example); (2) the size of the electron edge depends on a combination of many parameters, so a very high correlation coefficient between the electron edge size and any one of the parameters is not likely.

Panel (f) of Fig. 8 shows how the size of the electron edge depends on the magnetic local time of observation. There is no clear correlation or dependency between these two data sets. However, for MLT dependency, the linear least squares fit and Pearson correlation coefficient may not be the best way to analyse the data, as we would expect some symmetrical changes in the LLBL electron edge size from the noon sector in both the dusk and dawn directions, similar to the LLBL size itself (Newell and Meng, 1992). We thus re-organized the MLT data as a departure from 12:00 MLT, and re-plotted the electron edge size versus this re-organised data and performed a similar analysis. The results are shown in Fig. 9, panel (a). There is a slight increase in the LLBL electron edge size with MLT time difference from the local noon. It also seems possible that the number of events of smaller size, $0-0.3^{\circ}$ ILAT, is higher near the 12:00 MLT sector. However, the correlation coefficient between the LLBL electron edge size and MLT time shift from the local noon is very low, $C C=0.04$ as is the statistical significance of result, $S S=35 \%$ (see Table 2).

Returning to panels $(g-j)$ of Fig. 8, we note these show poor correlations and very low statistical significances of results: the correlation coefficient varied in the range $C C=0.01-0.09$ (see Table 1) and the statistical significance was $S S=9-69 \%$. Furthermore, we do not find any simple dependency in the median values in the bins for these parameters, except for the IMF $B_{Y}$-component (panel h). For the 

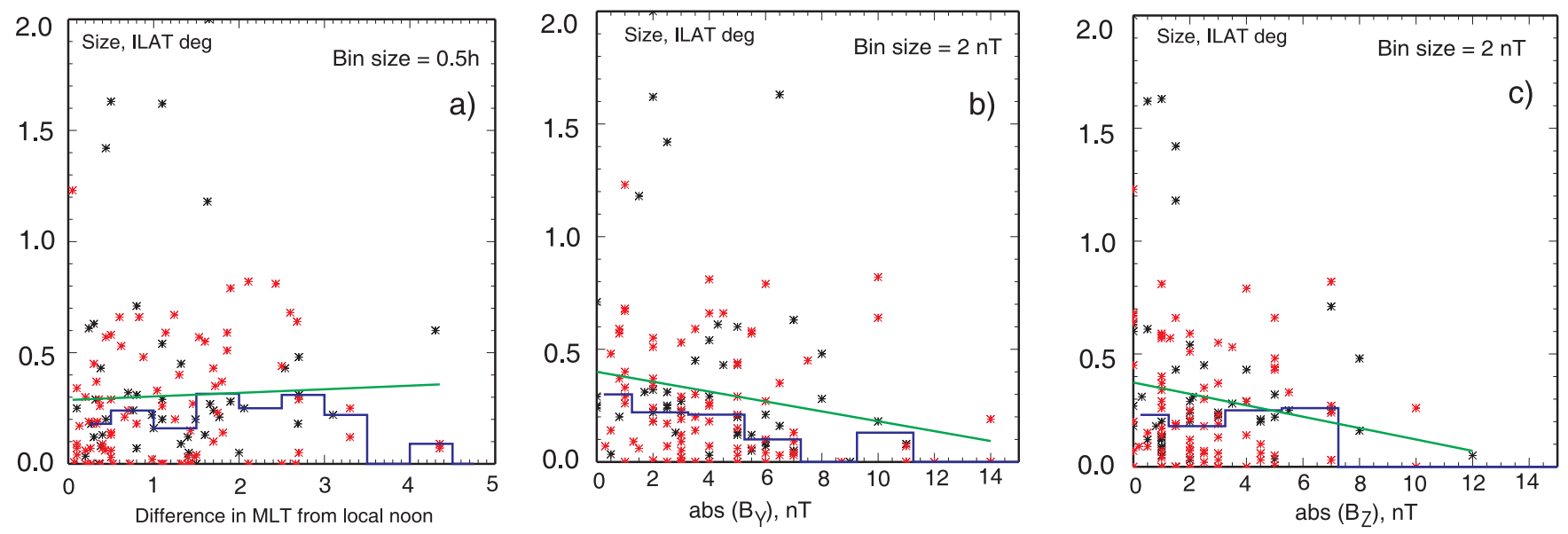

Fig. 9. Dependencies of the LLBL electron edge size from: the MLT difference from the local noon (panel a), the absolute value of the IMF $B_{Y}$-component (panel b) and the IMF $B_{Z}$-component (panel c). The GSM coordinate system has been used. In each panel, the red points represent events where the size was estimated from the multi-spacecraft method, while the black points represent events where the size was estimated from the one-spacecraft method. The green line shows a linear least squares fit to these data, while the blue line shows the median value of the size in each bin, for cases where there are more than 3 points in a bin.

IMF $B_{Y}$-component, there is an obvious trend in the median of the bin values, suggesting that size of the electron edge may be higher for $B_{Y} \sim 0$. Given this, we have checked how the LLBL electron edge size depends on absolute values of the IMF components. Panels (b) and (c) of Fig. 9 show how the LLBL electron edge size depends on the absolute values of the IMF $B_{\mathrm{Y}}$-component and $B_{\mathrm{Z}}$-component, respectively. Similar to Fig. 8, the red points represent events where the size was estimated from the multi-spacecraft method, while the black points represent events where the size was estimated from the one-spacecraft method. The green line shows a linear least square fit to these data and the blue line shows the median value of the size in each bin, for cases where there are more than 3 points in the bin. Estimated correlation coefficients and statistical significances of correlation between the LLBL electron edge size and absolute values of the IMF $B_{\mathrm{Y}^{-}}, B_{\mathrm{Z}^{-}}$, and $B_{\mathrm{X}}$-components are presented in the Table 2. There is an obvious trend between median values of the size of the LLBL electron edge and absolute value of the $B_{Y}$-component of IMF. The correlation coefficient between these two values is relatively high, $C C=-0.185$, and this result is statistically significant, $S S=96.3 \%$. A trend between the LLBL electron edge size median values and the absolute value of the IMF $B_{\mathrm{Z}}$-component is also evident (Fig. 9, panel c), however, the correlation coefficient was a little lower, $C C=-0.166$ and this result had lower statistical significance, $S S=93.98 \%$. Finally, we find no trend between the LLBL electron edge size and the absolute value of the IMF $B_{X}$-component (not shown) and correlation between these two parameters was very small, $C C=-0.02$ and $S S=12 \%$.
In summary, there are only two results with $S S>95 \%$ (correlation between the electron edge size and magnitude of the IMF as well as absolute value of the IMF $B_{Y}$-component) which can be considered as statistically significant. However, there are four distinct trends between median values of the electron edge size and the invariant latitude, ILAT, of observation, the level of geomagnetic activity $K_{\mathrm{p}}$, the solar wind dynamic pressure $P_{\mathrm{sw}}$, and the absolute value of the IMF $B_{\mathrm{Z}}$-component. Such trends in the median values could suggest a link between the LLBL electron edge size and these four external parameters (we note trends in the median values have been used before in statistical cusp studies (e.g., Zhou et al., 2000; Russell, 2000). In addition, we believe that there is slight trend between the median values of the LLBL electron edge size and how far from the local noon observations were made: it seems that the size of the electron edge possibly increases with the distance from the local noon. For other parameters we do not find significant correlations or any possible dependencies in the median values. A similar analysis has been performed on the database containing only the multi-spacecraft estimation of the boundary layer size and small distances between spacecraft (to increase accuracy). For these events (38 events) the correlation coefficients between the electron edge size and different external parameters slightly increased, but the statistical significance of results decreased due to the smaller data set. 


\section{Discussion}

5.1 Discussion of the plasma population inside the electron edge of the LLBL and comparison with previous observations

In Sect. 3 we presented plasma data for the mid-altitude cusp crossing which exhibited a distinct electron edge near the equatorward boundary of the LLBL/cusp. The presented solar wind and IMF data indicate external conditions favourable for the dayside magnetopause reconnection. Using the low-energy cut-offs in the precipitating and mirrored magnetosheath-like populations, it is possible to estimate the distance along the field line to the reconnection site at the magnetopause (e.g. Onsager et al., 1991; Fuselier et al., 2000; Trattner et al., 2004). The distance to the reconnection line $X_{\mathrm{r}}$ is defined as $X_{\mathrm{r}} / X_{\mathrm{m}}=2 V_{\mathrm{e}} /\left(V_{\mathrm{m}}-V_{\mathrm{e}}\right)$. In this relation, $X_{\mathrm{m}}$ is the distance to the ionospheric mirror point, $V_{\mathrm{e}}$ is the cutoff velocity of the precipitating ions, and $V_{\mathrm{m}}$ is the cut-off velocity of the mirrored distribution. We apply this method (for a fuller description see Trattner et al., 2004) in order to estimate the distance to the reconnection point during this cusp crossing. Using the cut-offs observed at a number of distinct times, we obtain results for the distance to the reconnection site for this event which varied in the range $18-24 R_{E}$. This is consistent with the expected position of the reconnection site on the dayside magnetopause. The time delay between observations of the electron boundary of the LLBL/cusp and ion boundary, where $2 \mathrm{keV}$ ions have been detected, is $220 \mathrm{~s}$. This time delay between the arrival of electrons and energetic ions agrees very well with an estimated average field-aligned distance of $\sim 20-21 R_{\mathrm{E}}$ between Cluster and the reconnection site. This supports our conclusion that LLBL electron edge is formed on the newly-reconnected field lines.

The electron edge of the LLBL contains a "background" of fairly isotropic electrons with low flux and density. The density is around $10-20 \%$ of that observed later in the cusp proper. We suppose therefore that this electron distribution corresponds to the suprathermal halo part of the solar wind electrons, as suggested and modelled by Wing et al. (1996) and Wing et al. (2005). Inside the electron edge, the electron anisotropy was quite low, $T_{\|} / T_{\perp}<1.1$, most of the time. This event contrasts with a different type of the LLBL electron edge event discussed in Bogdanova et al. (2004). During their event, on 23 August 2001, 12:00-13:20 UT, the electron edge of the LLBL was observed for $2 \mathrm{~min}$ and, in addition to the background low-density electrons, uni- or bi-directional electron beams (suprathermal electron bursts) were detected inside the LLBL electron edge (see Fig. 1 from Bogdanova et al., 2004) with electron anisotropy $T_{\|} / T_{\perp}>1$.2. In that case, the downgoing electron beams may originate near the dayside X-line during active reconnection, as predicted by reconnection theory (e.g. Sibeck et al., 1999) and observed near the magnetopause (e.g. Gosling et al., 1990). The most likely source of the upgoing short duration electron beams inside the electron edge is a wave-particle interaction process. For example, it has been suggested that electrons could be trapped in the parallel electric fields associated with kinetic Alfvén waves and propagate with these waves (Chaston et al., 2005). A parallel electric field existing below the spacecraft and accelerating electrons is also a possible explanation (e.g., Paschmann et al., 2003). The investigation of what physical parameters define the absence or existence of the electron beams inside the LLBL electron edge is a topic for future study.

Inside the electron edge, the ion population remains typical of the dayside plasma sheet as ions need longer times to propagate along the open field lines. At $\sim 13: 52$ UT the appearance of low energy, $E=30-100 \mathrm{eV}, \mathrm{O}^{+}$ions was detected. Appearance of the low-energy ions correlates with the enhancement of the electron anisotropy and the existence of anti-parallel low-energy electron beams. Apart of this short interval, no other ionospheric ion outflow was detected inside the LLBL electron edge. The local heating and outflow of both low-energy $\mathrm{H}^{+}$and $\mathrm{O}^{+}$ions were detected inside the LLBL proper, 13:54-13:55 UT, in correlation with a strong enhancement of electron anisotropy up to $\sim 1.2$. Absence of local ion heating and outflow inside the LLBL electron edge is in disagreement with previous studies of plasma properties inside this region (Topliss et al., 2001; Bogdanova et al., 2004). Both these studies reported strong local ion heating and outflow inside the LLBL electron edge. It was shown that local ion heating and the beginning of the outflow were very well correlated with suprathermal electron beams and it was suggested (Bogdanova et al., 2004) that injections of the electron beams with anisotropy more than 1.2 define the local ion heating and outflow of the ionospheric ions. The event discussed in this paper is consistent with such a conclusion as no ionospheric ion heating and outflow were observed inside the fairly isotropic LLBL electron edge in the absence of suprathermal electron bursts.

It is interesting to note that despite the fact that we see newly-reconnected field lines inside the LLBL electron edge, the plasma convection was very low. Similarly low levels of convection inside the electron edge were observed at ionosphere level by EISCAT in previous studies (e.g., Moen et al., 2004). Low convection inside the electron edge may be due to the finite velocity of the Alfvén waves that are responsible for the transmission of the magnetic stress from the reconnection site to the ionosphere (e.g., Moen et al., 2004; Farrugia et al., 2004; Cerisier et al., 2005). Enhancement of the anti-sunward plasma convection was observed from $\sim 12: 54$ UT, inside the LLBL proper and cusp region. The simultaneous observations of the convection enhancement and the arrival of the LLBL ions are in agreement with the Lockwood model on the interior Alfvén wave (Lockwood et al., 1996).

To summarise, the LLBL electron edge is characterised by the low-density, fairly isotropic magnetosheath-like electrons with or without electron beams, by the plasma sheet 
ions with or without low-energy, locally heated and outflowing ionospheric ions and by the low convection speed of the plasma. It was suggested (Wing et al., 1996), that a $\sim 250 \mathrm{~V}$ potential retards core populations of the solar wind electrons equatorward of the cusp. With the multi-point measurements from Cluster we have a unique opportunity to determine the efficiency of the such parallel electric field, which may exist between the magnetopause and low-altitudes, and which prevents the core population of solar wind electrons penetrating unimpeded along newly opened field lines to low-altitudes. In the future we intend to study other events inside the midaltitude LLBL/cusp with significant altitude difference between two or more Cluster spacecraft.

\subsection{Comparison of statistical results with previous studies}

Our statistical study shows that the electron edge of the LLBL is often observed during mid-altitude cusp crossings by Cluster: it has been observed in $87 \%$ of cusp crossings where clear electron and ion boundaries have been identified or in $\sim 35 \%$ of cusp crossings available for this study (including events with unclear boundaries). It is interesting to compare our results with statistical results from Newell and co-workers (Newell and Meng, 1988, 1992; Newell et al., 1991a,b) who performed a large statistical survey and created the well-known map of the different magnetospheric regions as seen at low-altitudes (Newell and Meng, 1992). The electron edge of the LLBL described here was not included in their statistical study. However, they discussed a region named the Boundary Plasma Sheet (BPS), which is a region with soft (magnetosheath-like) electrons in the dawn and dusk sectors. Newell et al. (1991b) and Newell and Meng (1992) described the BPS as any region of soft electron precipitation (except polar rain) which does not fall into other categories (cusp, mantle and LLBL). It was pointed out that usually this region resembles the nightside BPS, i.e. ion precipitation shows no clear sign of a magnetosheath origin, electron temperatures are a few hundred $\mathrm{eV}$ and spatially and spectrally structured electron spectra are common. Newell and Meng (1992) regarded the dayside BPS as an extension of nightside BPS, implying that the ions are accelerated on newly closed field lines in the tail. However, Lockwood (1997) noted that there are usually continuous energy-latitude dispersions in the ion population inside the BPS/LLBL and cusp regions, and explained both the BPS and LLBL ions in the terms of the open magnetosphere model. Another region within Newell and Meng (1992) classification was termed "void" which was observed between the Central Plasma Sheet (CPS) and the LLBL and consists of low-flux plasma. Newell and Meng defined the void, or photoelectron region, by the sharp flux drop-off above $\sim 70 \mathrm{eV}$. Lockwood (1997) and Onsager and Lockwood (1997) argued that this void region can occur when and where the flux falls below the instrument one-count level, which depends on the instrument geometric factor (and thus its sensitivity threshold). Thus they re-classified the void region near noon (between CPS and LLBL) as being part of the dayside BPS.

The plasma properties inside the Newell and Meng "void" and "BPS" regions and the Lockwood "BPS" region are similar to those observed by Cluster inside the electron edge of the LLBL. We therefore suggest that at least part of the void or/and BPS regions seen in the Newell and Meng statistics and the BPS region discussed by Lockwood corresponds to the electron edge of the LLBL seen in the Cluster data. The magnetosheath-like electrons suggest that this boundary layer is on open field lines and places it poleward of the OCB. This conclusion is in agreement with the suggestion that the LLBL electron edge will be near the CPS-BPS border in the open magnetosphere model (e.g., Lockwood, 1997). In this case, the term "electron edge of the LLBL" is perhaps a more topologically appropriate name than "Boundary Plasma Sheet" as it implies the connection to dayside processes.

Topliss et al. (2001) studied a similar electron edge observed in Polar data. Their statistical study shows the existence of this boundary layer in only 6 events out of 200 mid-altitude cusp crossings. This result is very different from our result where we observed an electron edge of the LLBL in $87 \%$ of the mid-altitude cusp crossings with clear boundaries or in $35 \%$ of all cusp crossings used in this study. A number of possible reasons could explain this discrepancy: (1) Topliss et al. did not describe the criteria used for the definitions of the electron and ion boundaries. It is possible that they used different definitions for clear electron and ion boundaries, which might lead to a slightly different result; (2) Topliss et al. did not mention how many events they have with clear ion and electron boundaries. In the Cluster data we have only $\sim 40 \%$ events out of all crossings in which boundaries could be clearly defined. It is possible that Topliss et al. analysed many events with unclear ion and electron boundaries; (3) As discussed above, the sensitivity of instruments is essential in the accurate detection of the electron edge, so it is possible that the Polar spacecraft has a different sensitivity; (4) The time resolution of the Polar TIMAS particle instrument (with full energy range coverage) is $12 \mathrm{~s}$ (Shelley et al., 1995) compared to $4 \mathrm{~s}$ for Cluster, so it is possible that events with a time difference between two boundaries less than $12 \mathrm{~s}$ were not recognisable in the Polar data; (5) Polar sampled mostly in the noon sector. Cluster observations also show that near noon there are many events with simultaneous electron and ion boundaries. Another possible explanation is that during the Polar observations the parallel electric field was stronger and the suprathermal part of the solar wind electrons was also retarded, as suggested in Topliss et al. (2001). Topliss et al presented LLBL/cusp observations during the minimum of the solar cycle 22, during years 1996-1998; however Cluster observations were done during maximum of the following solar cycle 23, during years 2001-2003. We suggest that probably we see solar cycle dependency in 
our results: it is possible that the suprathermal halo population of the solar wind is more dense or more energetic during the solar maximum and hence penetrate deeper into the Earth's magnetosphere or/and flux of the halo population is higher than one-count level and hence could be easily detected by the electron spectrometer in comparison with less dense or less energetic halo population during the solar minimum. Studies of the variability of the solar wind during the solar cycle (e.g., Stamper et al., 1999; Holzer, 2005) show that in general a higher speed and lower density solar wind is observed during solar minimum in comparison with a lower speed and higher density solar wind during solar maximum. We suggest that the halo part of the solar wind could inhibit the similar dependencies.

We propose that some combination of the six factors discussed above could possibly explain that in Cluster data the electron edge of the LLBL have been observed more frequently than in the Polar data.

\subsection{Position of the electron edge of the LLBL/cusp}

In this section we discuss how the position of the observed LLBL/cusp electron boundary depends on different parameters. The electron boundary can be used as a good approximation of the Open Closed Boundary as well as rough approximation of the equatorward boundary of the cusp itself and so it is also informative to compare our results with previous studies of the cusp position (Newell and Meng, 1988; Woch and Lundin, 1992; Aparicio et al., 1991; Merka et al., 2002; Zhou et al., 2000; Russell, 2000; Wing et al., 2001). As in previous studies the equatorward boundary of the cusp was identified from the ion or magnetic field data, we could not directly compare our results with their results. However, we showed (Sect. 4.6) that the median value of difference between the invariant latitudes of the electron and ion equatorward boundaries of the LLBL/cusp is $0.2^{\circ}$ ILAT. So, having this difference in the mind, we can compare our results with previous studies. Our observations were made under the typical solar wind conditions and IMF orientation favourable for reconnection at the dayside magnetopause equatorward of the cusps, as described in Sects. 4.4 and 5.1 (Fig. 5).

The electron boundary of the observed LLBL/cusp crossings by Cluster at mid-altitudes varied in the range of $69^{\circ}-$ $80^{\circ}$ ILAT, with most events having electron boundaries at $74^{\circ}-77^{\circ}$ ILAT (Fig. 5, panel a). This result partially agrees with Polar observations that the equatorward boundary of the polar cusp is located from $69^{\circ}$ to $87^{\circ}$ ILAT (Zhou et al., 2000). Note, in the present study we limited our database, and included only events with southward IMF or variable IMF. Thus we do not include observations of the cusp position for the strong northward IMF when poleward displacement of the cusp is likely.

The MLT of the electron boundary observed during the Cluster LLBL/cusp crossings spread in the range 09:0017:00 MLT, with the majority of events observed in the
10:00-15:00 MLT sectors (Fig. 5, panel b). The broad MLT location of the region agrees with the previous cusp studies (Zhou et al., 2000; Merka et al., 2002; Woch and Lundin, 1992; Kremser and Lundin, 1990). The maximum probability of the LLBL/cusp electron boundary observations in our study was at $\sim 12: 00$ MLT.

The dependency of the invariant latitude of the cusp on the IMF $B_{\mathrm{Z}}$-component is very well known (Zhou et al., 2000; Russell, 2000; Newell and Meng, 1988; Merka et al., 2002): the cusp will move equatorward with an increasing southward IMF component due to the magnetopause erosion (Farrugia et al., 2001; Yeoman et al., 2002; Karlson et al., 1996) and the cusp will be displaced poleward due to the highlatitude lobe reconnection (Dungey, 1963; Crooker, 1979). It was shown (Zhou et al., 2000) that there is almost linear dependency between ILAT of the equatorward boundary of the cusp and negative values of the IMF $B_{\mathrm{Z}}$-component. In our statistical study, we see (Fig. 6, left panel) a similar dependency of the ILAT of the electron boundary of the LLBL/cusp on IMF $B_{Z}$ as Zhou et al. (2000): the median values of the ILAT was $71.5^{\circ}$ under the IMF $B_{\mathrm{Z}} \sim-8 \mathrm{nT}$, and increased till $77^{\circ}$ ILAT under the IMF $B_{\mathrm{Z}} \sim-1 \mathrm{nT}$. However there is significant scatter in the individual observations, especially for the smaller values of the southward IMF, $-6 \mathrm{nT}<B_{\mathrm{Z}}<-0.5 \mathrm{nT}$. In recent years it was shown that the size of the polar cap, the position of the OCB at the dayside and hence the position of the cusp region depends on relative reconnection rates of low-latitude dayside magnetopause and nightside magnetotail reconnection processes (e.g., Cowley and Lockwood, 1992; Milan et al., 2005; Lockwood et al., 2005). For example, Lockwood et al. (2005) presented a study of the continuous motion of the dayside open-closed field line boundary during two substorm cycles, based on ionospheric data from the EISCAT VHF radar. In one event study, it was shown that the position of the OCB correlates with the auroral AL index and that OCB moves poleward during the expansion phase of a substorm, even for periods of the stable southward IMF. We suggest that the relative importance of the dayside and nightside reconnection rates could be the cause of the spread in the plot of IMF $B_{\mathrm{Z}}$ versus latitude of the electron edge. Under northward IMF the cusp does not move much and varied between $75^{\circ}-76^{\circ}$ ILAT under positive IMF $B_{Z}$, in agreement with previous studies (e.g., Zhou et al., 2000). However, recall that we consider events only with reconnection at the dayside magnetopause, so this result simply indicates that the reconnection rate at the dayside magnetopause is very small under northward IMF.

The dependency between the invariant latitude of the LLBL/cusp electron boundary and solar wind dynamic pressure is presented in the right panel of Fig. 6. There is a very clear trend in the median values: the invariant latitude of the LLBL/cusp electron boundary decreases with increasing solar wind pressure. Similar trends have been seen in highaltitude cusp crossings by Hawkeye (Eastman et al., 2000), where it was shown that the cusp moves equatorward by 
$0.2-0.3 R_{\mathrm{E}}$ for each $1 \mathrm{nPa}$ of pressure. However, this dependency has not been confirmed in other studies. Eastman et al. (2000) showed this dependency eliminating dipole tilt effect, splitting all events into two groups, with positive or negative dipole tilt angles. In this Cluster study, we did not split all events into different groups according to dipole tilt angle. However all events considered were in the northern hemisphere during late July-early October. Thus the tilt angle for all events was in the same direction, positive (summer) or close to zero (fall).

In summary, we find that the position of the electron boundary of the LLBL/cusp generally agrees with the previous studies of cusp position, as do the dependencies of the ILAT position of this boundary on the different external parameters.

5.4 The LLBL electron edge statistical properties - expectations and results

As discussed in Sect. 4.6, the size of the electron edge varies between $0^{\circ}$ and $2^{\circ}$ ILAT with a median value of $0.2^{\circ}$ ILAT. These values correspond very well to expectations from simple calculations based on plasma convection and distance to the magnetopause. Thus, Topliss et al. (2001) noted that for Polar observations, assuming the distance to the magnetopause reconnection site to be $10 R_{\mathrm{E}}$ and poleward convection speed inside the cusp to be $10-50 \mathrm{~km} \mathrm{~s}^{-1}$, the time difference between encountering electron and ion boundaries should be $3-10 \mathrm{~min}$. In our data the time difference between observations of these two boundaries varied between $10 \mathrm{~s}$ and 5 min.

With the Polar observations it was not possible to identify factors defining the size of the electron edge (Topliss et al., 2001). However, based on the larger Cluster data set, 327 events in total, we have found some dependencies. However, we note that the size of this boundary layer most likely depends on the combination of many external parameters. Indeed, even from simple considerations, the size of the electron edge as observed at mid-altitudes depends on at least (1) the relative position of the reconnection point and observation point, (2) the velocity of the ions marking the ion cusp/LLBL boundary and (3) convection speed of the reconnected field lines. The relative position of the reconnection point and observation point depends on the location of the magnetopause and the location of the X-line on the magnetopause. In turn, the former depends on the solar wind dynamic pressure (Chapman and Ferraro, 1931) and IMF $B_{Z^{-}}$ component (Shue et al., 1997). The location of the site of anti-parallel sub-solar reconnection could be shifted into the Southern or Northern hemispheres according to sign of the $B_{\mathrm{X}}$-component of the IMF (e.g. Sibeck et al., 1999). The location of the anti-parallel merging site also will be shifted into the dusk or dawn sectors according to the sign of the $B_{Y^{-}}$ component of IMF (e.g. Smith and Lockwood, 1996). Based on these assumptions, we would expect that the size of the electron edge will anti-correlate with the solar wind dynamic pressure and the $B_{\mathrm{Z}}$-component of the IMF. Observed in the northern hemisphere, the width of the electron edge should be larger for a negative IMF $B_{\mathrm{X}}$-component, and, when observed in the dusk (dawn) sector, the electron boundary layer size should be larger for the dawnward (duskward) IMF.

It was shown that the ions populated cusp region under southward IMF are accelerated at the dayside magnetopause to the local Alfvén velocity, $\mathbf{V}_{\mathbf{A}}=\mathbf{B} / \sqrt{\mu_{\mathbf{0}} \rho_{\mathbf{0}}}$, where $\boldsymbol{B}$ is the magnetic field strength, $\mu_{0}$ is the permeability of free space and $\rho_{0}$ is the mass density (e.g. Smith and Lockwood, 1996). Thus, the size of the region between the electron boundary of the LLBL and the arrival of the ions moving with local Alfvén velocity should anti-correlate with strength of the magnetic field and correlate with the plasma density.

The source of the energetic LLBL ions is still an open question. According to Lockwood et al. (1996) and Lockwood and Moen (1996), ions forming the LLBL region can be generated on open field lines by reflection of the preexisting magnetosphere population by an interior Alfvén wave. This wave is launched from the reconnection site into the inflow region, on the magnetospheric side of the boundary. It propagates faster away from the reconnection site than the exterior Alfvén wave, due to the smaller plasma density and the higher magnetic field inside the magnetosphere in comparison to the magnetosheath. Hence, the ions forming the LLBL will be accelerated to the local interior Alfvén velocity, which depends on magnetospheric magnetic field strength and plasma density. In this model, the cusp proper is formed by ions accelerated on the exterior Alfvén wave which stands in the magnetosheath and contains the major rotation of the magnetic field. Thus, the ions forming cusp region will be accelerated to the local exterior Alfvén velocity, which depends on the strength of the magnetosheath magnetic field and plasma density. However, Fuselier et al. (1999) suggested an alternative explanation of the energetic LLBL ions, based on the solar wind and magnetosheath ion distribution functions. They showed that the distribution of the magnetosheath ions already has $\sim 20 \%$ higher energy (hotter) ions and suggest that the LLBL forms due to the arrival of this high energy part of the magnetosheath distribution.

The convection of the reconnected field lines depends on the solar wind electric field (reconnection rate) (Smith and Lockwood, 1996; Lockwood and Smith, 1994; Lockwood et al., 1998; Sibeck et al., 1999). In this case, the size of the electron edge will correlate with the velocity of the solar wind and magnitude of the IMF. However, as discussed in Sect. 5.1, the information that a field line is reconnected and undergoes strong convection reaches the mid-altitude with some time delay corresponding to the time for Alfvén wave propagation from the reconnection point (e.g. Farrugia et al., 2004). Given this, the connection between the size of the electron edge and the reconnection rate is less clear. 
Thus there are at least six external parameters which should influence the size of the LLBL electron edge, and there may be many more (for example, the strength of the potential drop above the spacecraft) which could also influence the size of this boundary layer. Note that there is also a temporal factor in the electron edge size determination. If a spacecraft crosses into the cusp/boundary layer region, which has formed due to a reconnection pulse but some time after a reconnection pulse has ceased, it is possible that the spacecraft would not detect newly-reconnected field lines with only magnetosheath-like electrons, but would directly cross onto older reconnected field lines with both magnetosheath-like ions and electrons. At mid-altitudes, 4$7 R_{\mathrm{E}}$, the Cluster satellite speed in sunward-antisunward direction, $V_{\mathrm{X}} \sim 2-3 \mathrm{~km} \mathrm{~s}^{-1}$, is less than expected plasma convection speed inside the cusp, $V_{\mathrm{X}} \sim 10-50 \mathrm{~km} \mathrm{~s}^{-1}$. Thus, inside the cusp region, it is more likely that plasma will convect over spacecraft. However, inside the electron edge of the LLBL, convection speeds are very small and Cluster may cross the newly-reconnected field lines inside the LLBL electron edge by virtue of the spacecraft motion. In both scenarios, temporal variability of processes at the magnetopause could also influence the results.

Our statistical study shows that the size of the electron edge anti-correlates with the magnitude of the IMF as well as with the absolute values of the IMF $B_{Y}$ - and $B_{\mathrm{Z}}$-components. One possible explanation of such a dependency could be based on the suggestion that the ions which mark the ion boundary in our events propagate with an Alfvén velocity dependent on the magnetosheath parameters. In our dataset, the LLBL proper, containing a mixture of both high-energy, lowdensity electrons and ions, was sometimes missing or it was hard to distinguish between the cusp proper and the LLBL. We therefore believe that the ion boundary in many cases corresponds to the arrival of ions in the cusp proper, which have been accelerated by the exterior Alfvén wave with parameters calculated based on magnetosheath values of magnetic field and density (Lockwood et al., 1996; Lockwood and Moen, 1996). This could explain why size of the LLBL electron edge depends on the magnitude as well as absolute values of the IMF $B_{\mathrm{Y}}$ - and $B_{\mathrm{Z}}$-components.

In the trends of median values, we have found some anticorrelation between solar wind dynamic pressure and the size of the electron edge, which might be expected on the basis of the discussion above. The slight dependency of the electron edge size on ILAT and the index of the geomagnetic activity $K_{\mathrm{p}}$ seen in the median values trend appears to be a "secondary effect", as both of these parameters depend in turn on the solar wind and IMF conditions. Our study shows that size of the LLBL electron edge slightly increases with the local time difference from 12:00 MLT. This result is in agreement with Newell and Meng (1992) observation of the proper LLBL, where it was shown that size if the LLBL increases towards the flanks. We did not find any obvious dependencies in the electron edge size on IMF $B_{\mathrm{X}}$. We suggest that the influence of the IMF $B_{\mathrm{X}}$ component is too complicated to find any simple trend: while this component defines the position of the reconnection at the magnetopause, the magnitude of this component is still partially defines the magnetosheath Alfvén velocity. We also find no correlation between the LLBL electron edge size and the solar wind density or velocity. In general, we conclude that while some parameters (IMF magnitude, absolute values of the IMF $B_{\mathrm{Y}^{-}}$ and $B_{\mathrm{Z}}$-components, and solar wind dynamic pressure $P_{\mathrm{sw}}$ ) clearly influence the size of the electron edge as expected from the simple reconnection model (e.g. Smith and Lockwood, 1996), other anticipated correlations have not been confirmed in this study. We suggest that the combination of at least six different factors makes the determination of the role of different parameters within a such statistical study very complicated. For more fruitful analysis, we need to obtain enough events to be able to "fix" some of the external parameters and study how the electron edge size depends on the variation on free parameters. This study must remain for the future, as we need to extend our database to do it. However, we are able to conclude from the current work that the magnitudes of the total IMF, IMF $B_{\mathrm{Y}^{-}}$and $B_{\mathrm{Z}}$-components and the solar wind dynamic pressure are the main factors influencing the size of this boundary layer.

\section{Conclusions}

We present results of a statistical study of the electron edge of the LLBL observed by Cluster during mid-altitude cusp crossings:

(1) The electron edge consists of low-density, fairly isotropic electrons presumably from the halo population of the solar wind. Sometimes uni- or bi-directional electron beams with high fluxes are also observed inside this region. Inside this boundary layer there are ions of magnetospheric origin sometimes accompanied by ionospheric low-energy heated population. The convection of plasma inside this region as observed at the mid-altitudes is low.

(2) This electron edge has been observed in $87 \%$ of the mid-altitude LLBL/cusp crossings with clear equatorward electron and ion boundaries by Cluster or in $35 \%$ of all events studied, which is a major difference from the previous Polar result (Topliss et al., 2001).

(3) The LLBL electron boundary was observed between $68^{\circ}$ and $80^{\circ}$ invariant latitude (ILAT) and 10:00-17:00 MLT. Based on Cluster data, we show that the latitudinal location of the electron edge is influenced by the IMF $B_{\mathrm{Z}}$-component and the solar wind dynamic pressure.

(4) With Cluster 4-point measurements we have introduced a multi-spacecraft technique of estimation of the size of this boundary layer to increase accuracy of this estimation.

(5) The size of the electron edge varies between $0^{\circ}$ and $2.0^{\circ}$ ILAT with a median value of $0.2^{\circ}$ ILAT. 
(6) The size of the electron edge depends on the combination of many external parameters. We found statistically significant anti-correlations between the electron edge size and (1) the magnitude of the IMF and (2) the absolute value of the IMF $B_{Y}$-component. Distinct trends between median values of the electron edge size and the absolute value of the IMF $B_{\mathrm{Z}}$-component, the solar wind dynamic pressure, the invariant latitude, and the level of geomagnetic activity $K_{\mathrm{p}}$ have been also found. The size of the LLBL electron edge slightly increases with increasing MLT from local noon. We did not find any dependencies on other parameters, such as IMF $B_{\mathrm{X}}$-component, and solar wind density or velocity.

Acknowledgements. This work is supported in the UK by the UCL/MSSL Particle Physics and Astronomy Research Council Rolling Grant. We would like to thank the CDAWeb team for providing the level 2 ACE MAG and ACE Solar Wind Experiment data.

Topical Editor I. A. Daglis thanks two referees for their help in evaluating this paper.

\section{References}

Aparicio, B., Thelin, B., and Lundin, R.: The polar cusp from a particle point of view - A statistical study based on Viking data, J. Geophys. Res., 96, 14 023-14 031, 1991.

Bogdanova, Y. V., Fazakerley, A. N., Owen, C. J., Klecker, B., Cornilleau-Wehrlin, N., Grison, B., André, M., Cargill, P., Rème, H., Bosqued, J. M., Kistler, L. M., and Balogh, A.: Correlation between suprathermal electron bursts, broadband extremely low frequency waves, and local ion heating in the midaltitude cleft/low-latitude boundary layer observed by Cluster, J. Geophys. Res., 109, A12 226, doi:10.1029/2004JA010554, 2004.

Cerisier, J.-C., Marchaudon, A., Bosqued, J.-M., McWilliams, K., Frey, H. U., Bouhram, M., Laakso, H., Dunlop, M., Förster, M., and Fazakerley, A.: Ionospheric signatures of plasma injections in the cusp triggered by solar wind pressure pulses, J. Geophys. Res., 110, A8204, doi:10.1029/2004JA010962, 2005.

Chapman, S. and Ferraro, V. C.: A new theory of magnetic storms, Terr. Magn. Atmosph. Elec., 36, 171-186, 1931.

Chaston, C. C., Peticolas, L. M., Carlson, C. W., McFadden, J. P., Mozer, F., Wilber, M., Parks, G. K., Hull, A., Ergun, R. E., Strangeway, R. J., Andre, M., Khotyaintsev, Y., Goldstein, M. L., Acuña, M., Lund, E. J., Reme, H., Dandouras, I., Fazakerley, A. N., and Balogh, A.: Energy deposition by Alfvén waves into the dayside auroral oval: Cluster and FAST observations, J. Geophys. Res., 110, A2211, doi:10.1029/2004JA010483, 2005.

Cowley, S. W. H. and Lockwood, M.: Excitation and decay of solar wind-driven flows in the magnetosphere-ionosphere system, Ann. Geophys., 10, 103-115, 1992,

http://www.ann-geophys.net/10/103/1992/.

Crooker, N. U.: Dayside merging and cusp geometry, J. Geophys. Res., 84, 951-959, 1979.

Devore, J. L.: Probability and Statistics for Engineering and the Sciences, International Thomson Publishing, 1995.

Dungey, J. W.: Interplanetary Magnetic Field and the Auroral Zones, Phys. Rev. Lett., 6, 47-48, 1961.

Dungey, J. W.: The structure of the ionosphere, or adventures in velocity space, Geophysics: The Earth's Environment, 1963.
Eastman, T. E., Boardsen, S. A., Chen, S.-H., Fung, S. F., and Kessel, R. L.: Configuration of high-latitude and highaltitude boundary layers, J. Geophys. Res., 105, 23 221-23 238, doi:10.1029/1999JA900269, 2000.

Edwards, A. L.: The Correlation Coefficient, An Introduction to Linear Regression and Correlation, W. H. Freeman, San Francisco, CA, 1976.

Farrugia, C., Lund, E., Sandholt, P., Wild, J., Cowley, S., Balogh, A., Mouikis, C., Möbius, E., Dunlop, M., Bosqued, J., Carlson, C., Parks, G., Cerisier, J., Kelly, J., Sauvaud, J., and Rème, H.: Pulsed flows at the high-altitude cusp poleward boundary, and associated ionospheric convection and particle signatures, during a Cluster - FAST - SuperDARN- Søndrestrøm conjunction under a southwest IMF, Ann. Geophys., 22, 2891-2905, 2004, http://www.ann-geophys.net/22/2891/2004/.

Farrugia, C. J., Mühlbachler, S., Biernat, H. K., and Torbert, R. B.: Dayside erosion during intervals of tenuous solar wind, J. Geophys. Res., 106, 25 517-25 528, doi:10.1029/2001JA000087, 2001.

Fuselier, S. A., Lockwood, M., Onsager, T. G., and Peterson, W. K.: The source population for the cusp and cleft/LLBL for southward IMF, Geophys. Res. Lett., 26, 1665-1668, doi:10.1029/1999GL900354, 1999.

Fuselier, S. A., Petrinec, S. M., and Trattner, K. J.: Stability of the high-Latitude reconnection site for steady northward IMF, Geophys. Res. Lett., 27, 473-476, 2000.

Gosling, J. T., Thomsen, M. F., Bame, S. J., Onsager, T. G., and Russell, C. T.: The electron edge of the low latitude boundary layer during accelerated flow events, Geophys. Res. Lett., 17, 1833-1836, 1990.

Gustafsson, G., André, M., Carozzi, T., Eriksson, A. I., Fälthammar, C.-G., Grard, R., Holmgren, G., Holtet, J. A., Ivchenko, N., Karlsson, T., Khotyaintsev, Y., Klimov, S., Laakso, H., Lindqvist, P.-A., Lybekk, B., Marklund, G., Mozer, F., Mursula, K., Pedersen, A., Popielawska, B., Savin, S., Stasiewicz, K., Tanskanen, P., Vaivads, A., and Wahlund, J.-E.: First results of electric field and density observations by Cluster EFW based on initial months of operation, Ann. Geophys., 19, 1219-1240, 2001, http://www.ann-geophys.net/19/1219/2001/.

Heikkila, W. J. and Winningham, J. D.: Penetration of magnetosheath plasma to low altitudes through the dayside magnetospheric cusps, J. Geophys. Res., 76, 883-891, 1971.

Holzer, T. E.: Heating and Acceleration of the Solar Plasma (Tutorial Talk), in: ESA SP-592: Solar Wind 11/SOHO 16, Connecting Sun and Heliosphere, 2005.

Johnstone, A. D., Alsop, C., Burge, S., Carter, P. J., Coates, A. J., Coker, A. J., Fazakerley, A. N., Grande, M., Gowen, R. A., Gurgiolo, C., Hancock, B. K., Narheim, B., Preece, A., Sheather, P. H., Winningham, J. D., and Woodliffe, R. D.: Peace: a Plasma Electron and Current Experiment, Space Sci. Rev., 79, 351-398, 1997.

Karlson, K. A., Øieroset, M., Moen, J., and Sandholt, P. E.: A statistical study of flux transfer event signatures in the dayside aurora: The IMF By-related prenoon-postnoon asymmetry, J. Geophys. Res., 101, 59-68, doi:10.1029/95JA02590, 1996.

Kremser, G. and Lundin, R.: Average spatial distributions of energetic particles in the midaltitude cusp/cleft region observed by Viking, J. Geophys. Res., 95, 5753-5766, 1990. 
Lockwood, M.: Relationship of dayside auroral precipitations to the open-closed separatrix and the pattern of convective flow, J. Geophys. Res., 102, 17 475-17 488, doi:10.1029/97JA01100, 1997.

Lockwood, M. and Moen, J.: Ion populations on open field lines within the dayside low-latitude boundary layer: theory and observations during a transient event, Geophys. Res. Lett., 23, 2895-2898, doi:10.1029/96GL02761, 1996.

Lockwood, M. and Smith, M. F.: Low and middle altitude cusp particle signatures for general magnetopause reconnection rate variations. 1: Theory, J. Geophys. Res., 99, 8531-8553, 1994.

Lockwood, M., Cowley, S. W. H., and Onsager, T. G.: Ion acceleration at both the interior and exterior Alfvén waves associated with the magnetopause reconnection site: Signatures in cusp precipitation, J. Geophys. Res., 101, 21 501-21 514, doi:10.1029/96JA01948, 1996.

Lockwood, M., Davis, C. J., Onsager, T. G., and Scudder, J. D.: Modelling signatures of pulsed magnetopause reconnection in cusp ion dispersion signatures seen at middle altitudes, Geophys. Res. Lett., 25, 591-594, doi:10.1029/98GL00185, 1998.

Lockwood, M., Moen, J., van Eyken, A. P., Davies, J. A., Oksavik, K., and McCrea, I. W.: Motion of the dayside polar cap boundary during substorm cycles: I. Observations of pulses in the magnetopause reconnection rate, Ann. Geophys., 23, 3495-3511, 2005, http://www.ann-geophys.net/23/3495/2005/.

McCall, R. B.: Fundamental Statistics for the Behavioral Sciences, Harcourt Brace Jovanovich, New York, 1990.

Merka, J., Safránková, J., and Nemecek, Z.: Cusp-like plasma in high altitudes: a statistical study of the width and location of the cusp from Magion-4, Ann. Geophys., 20, 311-320, 2002, http://www.ann-geophys.net/20/311/2002/.

Milan, S. E., Hubert, B., and Grocott, A.: Formation and motion of a transpolar arc in response to dayside and nightside reconnection, J. Geophys. Res., 110, A1212, doi:10.1029/2004JA010835, 2005.

Moen, J., Carlson, H. C., and Sandholt, P. E.: Continuous observation of cusp auroral dynamics in response to an IMF By polarity change, Geophys. Res. Lett., 26, 1243-1246, doi:10.1029/1999GL900224, 1999.

Moen, J., Lockwood, M., Oksavik, K., Carlson, H., Denig, W., Eyken, A., and McCrea, I.: The dynamics and relationships of precipitation, temperature and convection boundaries in the dayside auroral ionosphere, Ann. Geophys., 22, 1973-1987, 2004, http://www.ann-geophys.net/22/1973/2004/.

Newell, P. T. and Meng, C.-I.: The cusp and the cleft/boundary layer - Low-altitude identification and statistical local time variation, J. Geophys. Res., 93, 14 549-14 556, 1988.

Newell, P. T. and Meng, C.-I.: Mapping the dayside ionosphere to the magnetosphere according to particle precipitation characteristics, Geophys. Res. Lett., 19, 609-612, 1992.

Newell, P. T., Meng, C.-I., Sanchez, E. R., Burke, W. J., and Greenspan, M. E.: Identification and observations of the plasma mantle at low altitude, J. Geophys. Res., 96, 35-45, 1991a.

Newell, P. T., Sanchez, E. R., Meng, C.-I., Burke, W. J., Greenspan, M. E., and Clauer, C. R.: The low-latitude boundary layer and the boundary plasma sheet at low altitude - Prenoon precipitation regions and convection reversal boundaries, J. Geophys. Res., 96, $21013-21023,1991 b$.

Onsager, T. G. and Lockwood, M.: High-Latitude Particle Precipitation and its Relationship to Magnetospheric Source Regions,
Space Sci. Rev., 80, 77-107, 1997.

Onsager, T. G., Thomsen, M. F., Elphic, R. C., and Gosling, J. T.: Model of electron and ion distributions in the plasma sheet boundary layer, J. Geophys. Res., 96, 20 999-21 011, 1991.

Onsager, T. G., Chang, S.-W., Perez, J. D., Austin, J. B., and Janoo, L. X.: Low-altitude observations and modeling of quasi-steady magnetopause reconnection, J. Geophys. Res., 100, 1883118 843, 1995.

Paschmann, G., Haaland, S., and Treumann, R.: Auroral Plasma Physics, Kluwer Academic Publishers, Dordrecht, Dordrecht, 2003.

Rème, H., Aoustin, C., Bosqued, J. M., Dandouras, I., Lavraud, B., Sauvaud, J. A., Barthe, A., Bouyssou, J., Camus, T., Coeur-Joly, O., Cros, A., Cuvilo, J., Ducay, F., Garbarowitz, Y., Medale, J. L., Penou, E., Perrier, H., Romefort, D., Rouzaud, J., Vallat, C., Alcaydé, D., Jacquey, C., Mazelle, C., D’Uston, C., Möbius, E., Kistler, L. M., Crocker, K., Granoff, M., Mouikis, C., Popecki, M., Vosbury, M., Klecker, B., Hovestadt, D., Kucharek, H., Kuenneth, E., Paschmann, G., Scholer, M., Sckopke, N., Seidenschwang, E., Carlson, C. W., Curtis, D. W., Ingraham, C., Lin, R. P., McFadden, J. P., Parks, G. K., Phan, T., Formisano, V., Amata, E., Bavassano-Cattaneo, M. B., Baldetti, P., Bruno, R., Chionchio, G., di Lellis, A., Marcucci, M. F., Pallocchia, G., Korth, A., Daly, P. W., Graeve, B., Rosenbauer, H., Vasyliunas, V., McCarthy, M., Wilber, M., Eliasson, L., Lundin, R., Olsen, S., Shelley, E. G., Fuselier, S., Ghielmetti, A. G., Lennartsson, W., Escoubet, C. P., Balsiger, H., Friedel, R., Cao, J.-B., Kovrazhkin, R. A., Papamastorakis, I., Pellat, R., Scudder, J., and Sonnerup, B.: First multispacecraft ion measurements in and near the Earth's magnetosphere with the identical Cluster ion spectrometry (CIS) experiment, Ann. Geophys., 19, 1303-1354, 2001 ,

http://www.ann-geophys.net/19/1303/2001/.

Russell, C. T.: Polar Eyes the Cusp, in: Cluster-II Workshop: Multiscale/Multipoint Plasma Measurements, Proceedings of the Workshop held at Imperial College, London, UK, 22-24 September 1999. Paris: European Space Agency (ESA), 2000, ESA-SP, vol. 449, ISBN: 9290927968, p.47, edited by: Harris, R. A., pp. 47-55, 2000.

Sandholt, P. E., Denig, W. F., Farrugia, C. J., Lybekk, B., and Trondsen, E.: Auroral structure at the cusp equatorward boundary: Relationship with the electron edge of low-latitude boundary layer precipitation, J. Geophys. Res., 107, SMP 10-1-SMP 10-9, doi:10.1029/2001JA005081, 2002.

Shelley, E. G., Ghielmetti, A. G., Balsiger, H., Black, R. K., Bowles, J. A., Bowman, R. P., Bratschi, O., Burch, J. L., Carlson, C. W., Coker, A. J., Drake, J. F., Fischer, J., Geiss, J., Johnstone, A., Kloza, D. L., Lennartsson, O. W., Magoncelli, A. L., Paschmann, G., Peterson, W. K., Rosenbauer, H., Sanders, T. C., Steinacher, M., Walton, D. M., Whalen, B. A., and Young, D. T.: The Toroidal Imaging Mass-Angle Spectrograph (TIMAS) for the Polar Mission, Space Sci. Rev., 71, 497-530, 1995.

Shue, J.-H., Chao, J. K., Fu, H. C., Russell, C. T., Song, P., Khurana, K. K., and Singer, H. J.: A new functional form to study the solar wind control of the magnetopause size and shape, J. Geophys. Res., 102, 9497-9512, doi:10.1029/97JA00196, 1997.

Sibeck, D. G., Paschmann, G., Treumann, R. A., Fuselier, S. A., Lennartsson, W., Lockwood, M., Lundin, R., Ogilvie, K. W., Onsager, T. G., Phan, T.-D., Roth, M., Scholer, M., Sckopke, N., 
Stasiewicz, K., and Yamauchi, M.: Chapter 5-Plasma Transfer Processes at the Magnetopause, Space Sci. Rev., 88, 207-283, doi:10.1023/A:1005255801425, 1999.

Smith, M. F. and Lockwood, M.: Earth's magnetospheric cusps, Rev. Geophys., 34, 233-260, doi:10.1029/96RG00893, 1996.

Stamper, R., Lockwood, M., Wild, M. N., and Clark, T. D. G.: Solar causes of the long-term increase in geomagnetic activity, J. Geophys. Res., 104, 28 325-28 342, 1999.

Stubbs, T. J., Cargill, P. J., Lockwood, M., Grande, M., Kellett, B. J., and Perry, C. H.: Extended cusp-like regions and their dependence on the Polar orbit, seasonal variations, and interplanetary conditions, J. Geophys. Res., 109, A9210, doi:10.1029/2003JA010163, 2004.

Topliss, S., Johnstone, A., Coates, A., Peterson, W. K., Kletzing, C. A., and Russell, C. T.: Charge neutrality and ion conic distributions at the equatorward electron edge of the midaltitude cusp, J. Geophys. Res., 106, 21 095-21 108, doi:10.1029/2000JA003032, 2001.

Trattner, K. J., Fuselier, S. A., and Petrinec, S. M.: Location of the reconnection line for northward interplanetary magnetic field, J. Geophys. Res., 109, A3219, doi:10.1029/2003JA009975, 2004.

Wing, S., Newell, P. T., and Onsager, T. G.: Modeling the entry of magnetosheath electrons into the dayside-ionosphere, J. Geophys. Res., 101, 13 155-13 168, doi:10.1029/96JA00395, 1996.
Wing, S., Newell, P. T., and Ruohoniemi, J. M.: Double cusp: Model prediction and observational verification, J. Geophys. Res., 106, 25 571-25 594, doi:10.1029/2000JA000402, 2001.

Wing, S., Newell, P. T., and Meng, C.-I.: Cusp Modeling and Observations at Low Altitude, Surv. Geophys., 26, 341-367, doi:10.1007/s10712-005-1886-0, 2005.

Woch, J. and Lundin, R.: Magnetosheath plasma precipitation in the polar cusp and its control by the interplanetary magnetic field, J. Geophys. Res., 97, 1421-1430, 1992.

Woch, J. and Lundin, R.: The low-latitude boundary layer at midaltitudes - Identification based on Viking hot plasma data, Geophys. Res. Lett., 20, 979-982, 1993.

Yeoman, T. K., Hanlon, P. G., and McWilliams, K. A.: A statistical study of the location and motion of the HF radar cusp, Ann. Geophys., 20, 275-280, 2002, http://www.ann-geophys.net/20/275/2002/.

Zhou, X. W., Russell, C. T., Le, G., Fuselier, S. A., and Scudder, J. D.: Solar wind control of the polar cusp at high altitude, J. Geophys. Res., 105, 245-252, doi:10.1029/1999JA900412, 2000. 